\title{
Mannich base-connected syntheses mediated by ortho-quinone methides
}

\author{
Petra Barta, Ferenc Fülöp and István Szatmári*
}

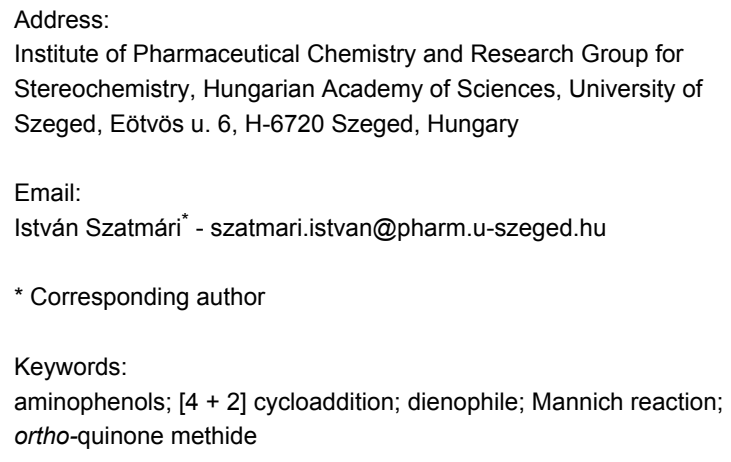

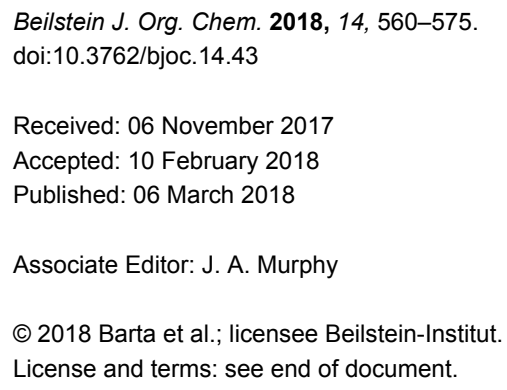

\section{Abstract}

This article provides an overview about specifically modified Mannich reactions where the process involves an ortho-quinone methide $(o-\mathrm{QM})$ intermediate. The reactions are classified on the basis of the $o$-QM source followed by the reactant, e.g., the dienophile partner in cycloaddition reactions $(\mathrm{C}=\mathrm{C}$ or $\mathrm{C}=\mathrm{N}$ dienophiles) or by the formation of multicomponent Mannich adducts. Due to the important pharmacological activities of these reactive $o$-QM intermediates, special attention is paid to the biological activity of these compounds.

\section{Review \\ Introduction}

The Mannich reaction is an important, one-pot, multicomponent, $\mathrm{C}-\mathrm{C}$ bond forming reaction that is widely used in the syntheses of many biologically active and natural compounds [1-5]. Originally, the Mannich product is formed through a three-component reaction containing a $\mathrm{C}-\mathrm{H}$ acid, formaldehyde and a secondary amine. Recently, one of its special variations called modified Mannich reaction, has gained ground, in which the $\mathrm{C}-\mathrm{H}$ acid is replaced by electron-rich aromatic compounds such as 1- and 2-naphthols as active hydrogen sources [6]. At the beginning of the 20th century, Mario Betti reported the synthesis of 1-aminobenzyl-2-naphthol starting from ammonia, benzaldehyde and 2-naphthol. This protocol is known as Betti reaction and the compound formed as Betti base [7-9]. Several examples have been published to extend the reaction and to synthesize varied substituted aminonaphthol derivatives [10]. Their relatively easy accessibility and promising biological properties have led to the resurgence of their chemistry coming again into the focus of pharmacological research.

The formation of aminonaphthols can be explained by two mechanisms. According to one possibility, first the reaction of the amine and the aldehyde yields a Schiff base and then the 
latter reacts with 2-naphthol in the second nucleophilic addition step. The other theory assumes the formation of an orthoquinone methide (o-QM) intermediate by the reaction of 2-naphthol and benzaldehyde. Re-aromatization, the driving force of the transformation, takes place in the second step by the nucleophilic addition of the amine component.

The class of $o-\mathrm{QMs}$ has recently been investigated from many aspects. They are known as short-lived species playing an important role as key intermediates in numerous synthetic pathways. Reviews have recently been published about $o$-QM generation, applicability in organic syntheses and biological properties [11-17]. However, in this review we would like to focus on their role in syntheses connected to Mannich base chemistry as well as their wider applicability and properties.

\section{Formation of Mannich bases via o-QM intermediates \\ Synthesis of amidoalkylnaphthols}

The preparation of amidoalkylnaphthols has recently been discussed from many points of view [18]. This indicates the importance of this reaction because 1-amidoalkyl-2-naphthols can be easily converted to important biologically active 1-aminoalkyl2-naphthol derivatives by a simple amide hydrolysis.

The mechanism of the Mannich reaction is depicted in Scheme 1. First, the reaction between the aldehyde and 2-naphthol, induced by the catalyst, leads to the generation of $o$-QM intermediate 3 that reacts further with the amide component to form the desired 1-amidoalkyl-2-naphthol derivatives. This second step can also be considered as a nucleophilic addition of the amide to the $o-\mathrm{QM}$ component.

Various catalysts and conditions were used to optimize reaction conditions considering economical and environmental aspects. These include microwave-assisted reactions, solvent-free conditions and the reusability of the catalyst (Table 1). Procedures are carried out as one-pot multicomponent transformations without the isolation of the intermediates formed. Therefore, with the application of nontoxic, readily available and inexpensive reagents, both time and energy are saved.

Recently the applicability of nanocatalysts in these reactions has been of interest since nanocatalysts, in general, are stable and recyclable and they exhibit higher activity than conventional catalysts. A few notable examples are worth mentioning here. Aluminatesulfonic acid nanoparticles (ASA NPs) proved to be efficient under neat conditions for the synthesis of 1-amidoalkyl-2-naphthols [19]. Zali et al. carried out this synthesis applying nano-sulfated zirconia [20], Borhade et al. used $\mathrm{PbS}$ nanoparticles [21], while Safari et al. applied magnetic-

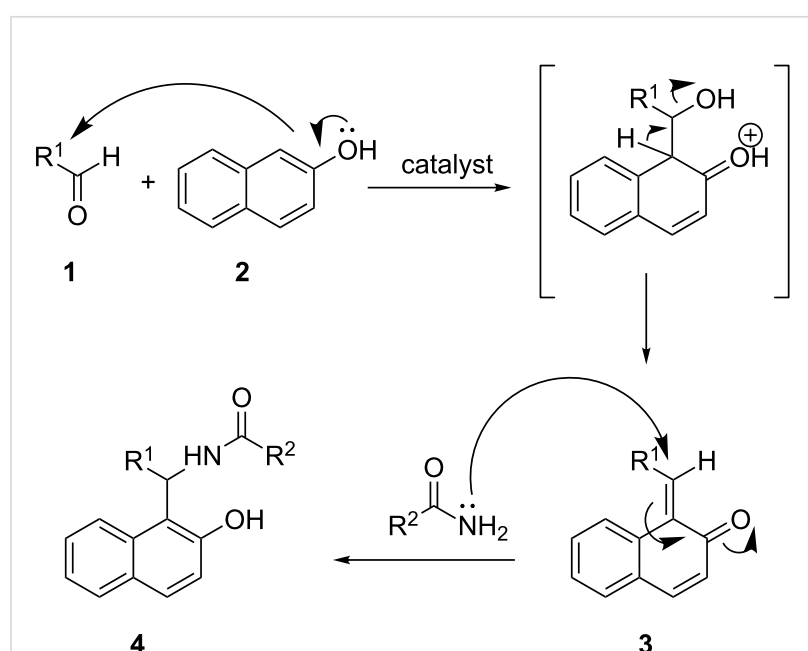

$R^{1}, R^{2}=$ see Table 1

Scheme 1: Formation of amidoalkylnaphthols 4 via o-QM intermediate 3.

nanoparticle-supported sulfuric acid ( $\left.\mathrm{MNPs}_{-} \mathrm{SO}_{3} \mathrm{H}\right)$ [22]. As shown in Table 1, entries 2-4, all methods give the desired amidoalkylnaphthols in $77-97 \%$ yields. Zolfigol et al. successfully applied 1-methylimidazolium tricyanomethanide $\left\{[\mathrm{HMIM}] \mathrm{C}(\mathrm{CN})_{3}\right\}$ as the first nanostructured molten salt [23]. As depicted in Table 1, entry 5, the catalyst gave remarkable results at room temperature in short reactions (5-30 minutes) in $90-96 \%$ yields. Comparing these results with those achieved by the application of tin dioxide nanoparticles (nano $\mathrm{SnO}_{2}$, Table 1, entry 6), molten salt catalysis affording higher yields in shorter reactions is definitely more advantageous.

Ionic liquids have also attracted considerable attention due to their ,green chemistry" values, including reusability, high thermal stability and non-inflammability. Hajipour et al. reported the one-pot synthesis of 1-amidoalkyl-2-naphthols catalysed by $N$-(4-sulfobutyl)triethylammonium hydrogen sulfate ([TEBSA][HSO 4$]$ ) as Brønsted acidic ionic liquid [24]. In addition, ethylammonium nitrate (EAN) [25], a sulfonic acid-functionalized benzimidazolium-based supported ionic liquid catalyst (SILC) [26], and carboxyl-functionalized benzimidazoliumbased ionic liquids (CFBILs) [27] proved to be efficient in the reaction (Table 1, entries $8-10$ ).

Safari et al. combined the benefits of using magnetic nanoparticles and ionic liquids by the application of magnetic $\mathrm{Fe}_{3} \mathrm{O}_{4}$ nanoparticles functionalized with 1-methyl-3-(3-trimethoxysilylpropyl)-1 $H$-imidazol-3-ium acetate (MNP-IL-OAc) as catalyst [28]. As shown in Table 1, entries 11 and 12, syntheses carried out by conventional heating at $100{ }^{\circ} \mathrm{C}$ required long reaction times affording yields of $82-97 \%$. In contrast, 
Table 1: Comparison of various conditions applied in the synthesis of amidoalkylnaphthols 4 .

\begin{tabular}{|c|c|c|c|c|c|c|}
\hline entry & $\mathrm{R}^{1}$ & $\mathrm{R}^{2}$ & catalyst & conditions & yields (\%) & ref. \\
\hline 1 & $\begin{array}{l}\mathrm{Ph}, 2,4-\mathrm{Cl}_{2} \mathrm{Ph}, 3-\mathrm{BrPh}, 4-\mathrm{NO}_{2} \mathrm{Ph} \\
\text { 4-(CHO)Ph, 5-Br-2-OHPh, 2-pyridyl, } \\
\text { 4-MePh, Et, 3-OEt-4-OHPh, 4-OHPh, } \\
\text { 4-OMePh, 3-BrPh }\end{array}$ & $\mathrm{Me}, \mathrm{Ph}, \mathrm{NH}_{2}$ & ASA NPs & $80^{\circ} \mathrm{C}, 8-30 \mathrm{~min}$ & $67-96$ & [19] \\
\hline 2 & $\begin{array}{l}\mathrm{Ph}, 2-\mathrm{CIPh}, 4-\mathrm{ClPh}, 4-\mathrm{BrPh}, 4-\mathrm{MePh} \\
\text { 4-OMePh, 3- } \mathrm{NO}_{2} \mathrm{Ph}, 4-\mathrm{NO}_{2} \mathrm{Ph}, 4-\mathrm{CNPh} \text {, } \\
\text { 3-OMePh, 1-Nph }\end{array}$ & $\mathrm{Me}, \mathrm{NH}_{2}$ & $\begin{array}{l}\text { nano-sulfated } \\
\text { zirconia }\end{array}$ & $120^{\circ} \mathrm{C}, 32-85 \mathrm{~min}$ & $81-94$ & {$[20]$} \\
\hline 3 & $\begin{array}{l}\mathrm{Ph}, 4-\mathrm{MePh}, 4-\mathrm{ClPh}, 4-\mathrm{OMePh} \\
\text { 4- } \mathrm{NO}_{2} \mathrm{Ph}, 3-\mathrm{NO}_{2} \mathrm{Ph}, 2-\mathrm{ClPh}, 2-\mathrm{NO}_{2} \mathrm{Ph} \text {, } \\
\text { 2-MePh, 3-OMePh }\end{array}$ & $\mathrm{Me}$ & PbS nanoparticles & $120^{\circ} \mathrm{C}, 4-9 \mathrm{~min}$ & $85-95$ & [21] \\
\hline 4 & $\begin{array}{l}\mathrm{Ph}, 4-\mathrm{MePh}, 4-\mathrm{NMe}_{2} \mathrm{Ph}, 4-\mathrm{OMePh}, \\
\text { 3-NO } \mathrm{NO}_{2} \mathrm{Ph}, 4-\mathrm{FPh}, 2,4-\mathrm{Cl}_{2} \mathrm{Ph}, \\
\text { 2,5-(OMe })_{2} \mathrm{Ph}, 3,4-(\mathrm{OMe})_{2} \mathrm{Ph}, \\
\text { 2,3-(OMe }{ }_{2} \mathrm{Ph}, 2-\mathrm{ClPh}, 2-\mathrm{NO}_{2} \mathrm{Ph} \text {, } \\
\text { 3-OH-4-OMePh, 2-FPh, } \mathrm{C}_{10} \mathrm{H}_{7} \text {, Et, } \mathrm{Pr}\end{array}$ & $\mathrm{Me}, \mathrm{Ph}, \mathrm{NH}_{2}$ & MNPs- $\mathrm{SO}_{3} \mathrm{H}$ & $100^{\circ} \mathrm{C}, 7-35 \mathrm{~min}$ & $77-97$ & {$[22]$} \\
\hline 5 & \multirow{2}{*}{$\begin{array}{l}\mathrm{Ph}, 4-\mathrm{NO}_{2} \mathrm{Ph}, 4-\mathrm{ClPh}, 2,5-(\mathrm{OMe})_{2} \mathrm{Ph} \text {, } \\
\text { 2-furyl, 2-thiophene, 1-Nph, 2-Nph, } \\
\mathrm{C}(\mathrm{Me})=\mathrm{CH}-\mathrm{Ph}, \mathrm{CH}=\mathrm{CH}-\mathrm{Ph}, \mathrm{Ph}-\mathrm{Ph}\end{array}$} & \multirow{2}{*}{$\mathrm{Me}, \mathrm{Ph}, \mathrm{NH}_{2}$} & $\left\{[\mathrm{HMIM}] \mathrm{C}(\mathrm{CN})_{3}\right\}$ & $\mathrm{rt}, 5-30 \mathrm{~min}$ & $90-96$ & [23] \\
\hline 6 & & & nano $\mathrm{SnO}_{2}$ & $\mathrm{rt}, 17-35 \mathrm{~min}$ & $81-88$ & [23] \\
\hline 7 & $\begin{array}{l}\mathrm{Ph}, 2-\mathrm{ClPh}, 4-\mathrm{ClPh}, 2,6-\mathrm{Cl}_{2} \mathrm{Ph}, 4-\mathrm{BrPh} \\
\text { 3- } \mathrm{NO}_{2} \mathrm{Ph}, 4-\mathrm{NO}_{2} \mathrm{Ph}, 3-\mathrm{MeOPh}, 4-\mathrm{MePh} \text {, } \\
\text { 2,5-(OMe) })_{2} \mathrm{Ph}, 4-\mathrm{CNPh}, 4-\mathrm{AcPh}\end{array}$ & $\mathrm{Me}, \mathrm{Ph}, \mathrm{NH}_{2}$ & {$[\mathrm{TEBSA}]\left[\mathrm{HSO}_{4}\right]$} & $120^{\circ} \mathrm{C}, 10 \mathrm{~min}$ & $73-91$ & [24] \\
\hline 8 & $\begin{array}{l}\mathrm{Ph}, 4-\mathrm{CIPh}, 4-\mathrm{OMePh}, 4-\mathrm{MePh}, 2 \text {-furyl, } \\
\text { 2-thiophene, 3-formylchromone }\end{array}$ & $\begin{array}{l}\mathrm{Me}, \mathrm{Ph}, \mathrm{NH}_{2} \text {, } \\
\text { OEt }\end{array}$ & EAN & $\mathrm{rt}, 60 \mathrm{~min}$ & $85-96$ & {$[25]$} \\
\hline 9 & $\begin{array}{l}\mathrm{Ph}, 3-\mathrm{NO}_{2} \mathrm{Ph}, 4-\mathrm{OHPh}, 4-\mathrm{OMePh} \\
2-\mathrm{CIPh}, 4-\mathrm{CIPh}, 4-\mathrm{NO}_{2} \mathrm{Ph}, 4-\mathrm{NMe}_{2} \mathrm{Ph} \\
3,4,5-(\mathrm{OMe})_{3} \mathrm{Ph}\end{array}$ & $\mathrm{Me}, \mathrm{Ph}, \mathrm{NH}_{2}$ & SILC & $100^{\circ} \mathrm{C}, 7-10 \mathrm{~min}$ & $80-95$ & {$[26]$} \\
\hline 10 & $\begin{array}{l}\mathrm{Ph}, 2-\mathrm{CIPh}, 4-\mathrm{ClPh}, 2-\mathrm{OMePh} \text {, } \\
\text { 4-OMePh, Et, } \mathrm{Pr}\end{array}$ & $\mathrm{Me}, \mathrm{Ph}, \mathrm{NH}_{2}$ & CFBILs & $70^{\circ} \mathrm{C}, 25-60 \mathrm{~min}$ & $84-94$ & {$[27]$} \\
\hline 11 & \multirow{2}{*}{$\begin{array}{l}\mathrm{Ph}, 4-\mathrm{MePh}, 4-\mathrm{OMePh}, 4-\mathrm{NMe}_{2} \mathrm{Ph}, \\
\text { 3- } \mathrm{NO}_{2} \mathrm{Ph}, 2,4-\mathrm{Cl}_{2} \mathrm{Ph}, 2-\mathrm{ClPh}, 2-\mathrm{NO}_{2} \mathrm{Ph} \text {, } \\
\text { 2,3-(OMe })_{2} \mathrm{Ph}, \mathrm{C}_{10} \mathrm{H}_{7}, \mathrm{Pr}\end{array}$} & \multirow{2}{*}{$\mathrm{Me}, \mathrm{Ph}, \mathrm{NH}_{2}$} & MNP-IL-OAC & $\begin{array}{l}100^{\circ} \mathrm{C} \\
60-210 \text { min }\end{array}$ & $82-97$ & [28] \\
\hline 12 & & & MNP-IL-OAC & $\begin{array}{l}\text { sonication, } \\
10-30 \mathrm{~min}\end{array}$ & $90-98$ & [28] \\
\hline 13 & \multirow{2}{*}{$\begin{array}{l}\mathrm{Ph}, 4-\mathrm{CIPh}, 3-\mathrm{NO}_{2} \mathrm{Ph}, 4-\mathrm{BrPh}, 4-\mathrm{MePh} \\
\text { 3- } \mathrm{NO}_{2} \mathrm{Ph}, 2-\mathrm{CIPh}, 2,4-\mathrm{Cl}_{2} \mathrm{Ph}\end{array}$} & \multirow{2}{*}{$\begin{array}{l}\mathrm{Me}, \mathrm{Ph}, \mathrm{NH}_{2} \\
\mathrm{NHMe} \text {, vinyl }\end{array}$} & $p$-TSA & DCE, rt, 9-30 h & $83-96$ & [29] \\
\hline 14 & & & $p$-TSA & $125^{\circ} \mathrm{C}, 4-10 \mathrm{~h}$ & $80-95$ & [29] \\
\hline 15 & $\begin{array}{l}\mathrm{Ph}, 2,4-\mathrm{Cl}_{2} \mathrm{Ph}, 3-\mathrm{OMePh}, 3-\mathrm{NO}_{2} \mathrm{Ph} \\
\text { 3,4-(OMe })_{2} \mathrm{Ph}, 4,5-(\mathrm{OMe})_{2}-2-\mathrm{NO}_{2} \mathrm{Ph} \text {, } \\
\text { 4-BrPh, 3,4,5-(OMe })_{3} \mathrm{Ph}, 2 \text {-pyridyl, } \\
\text { 3-indolyl, 2-furyl }\end{array}$ & $\mathrm{Me}, \mathrm{Ph}, \mathrm{NH}_{2}$ & $\begin{array}{l}\text { montmorillonite } \\
\text { K10 }\end{array}$ & $125^{\circ} \mathrm{C}, 30-120 \mathrm{~min}$ & $65-96$ & [30] \\
\hline 16 & $\begin{array}{l}\mathrm{Ph}, 3-\mathrm{NO}_{2} \mathrm{Ph}, 4-\mathrm{ClPh}, 4-\mathrm{BrPh}, 4-\mathrm{MePh} \\
\text { 4-FPh, 2-BrPh, 2-MePh, 2-CIPh, 3-BrPh }\end{array}$ & $\begin{array}{l}\mathrm{Me}, \mathrm{Ph}, \mathrm{NH}_{2} \\
\mathrm{NHMe} \text {, vinyl }\end{array}$ & Indion-130 & $110^{\circ} \mathrm{C}, 6-30 \mathrm{~min}$ & $81-94$ & [31] \\
\hline 17 & \multirow{2}{*}{$\begin{array}{l}\mathrm{Ph}, 4-\mathrm{MePh}, 4-\mathrm{ClPh}, 3-\mathrm{NO}_{2} \mathrm{Ph}, 2-\mathrm{BrPh} \\
2,4-\mathrm{Cl}_{2} \mathrm{Ph}, 1-\mathrm{Nph}, \mathrm{Et}\end{array}$} & \multirow{2}{*}{$\mathrm{Ph}, \mathrm{NH}_{2}, \mathrm{NHMe}$} & $\mathrm{I}_{2}$ & $\mathrm{DCE}, 125^{\circ} \mathrm{C}, 10-26 \mathrm{~h}$ & $35-93$ & [32] \\
\hline 18 & & & $\mathrm{I}_{2}$ & $125^{\circ} \mathrm{C}, 4-9 \mathrm{~h}$ & $20-90$ & [32] \\
\hline 19 & \multirow{2}{*}{$\begin{array}{l}\mathrm{Ph}, 4-\mathrm{BrPh}, 2-\mathrm{CIPh}, 4-\mathrm{CIPh}, 2,4-\mathrm{Cl}_{2} \mathrm{Ph} \\
\text { 3-OMePh, 3- } \mathrm{NO}_{2} \mathrm{Ph}, 4-\mathrm{MePh}\end{array}$} & \multirow{2}{*}{$\mathrm{Me}, \mathrm{Ph}, \mathrm{NH}_{2}$} & $\mathrm{~K}_{5} \mathrm{CoW}_{12} \mathrm{O}_{40} \cdot 3 \mathrm{H}_{2} \mathrm{O}$ & $125^{\circ} \mathrm{C}, 2-6 \mathrm{~h}$ & $74-88$ & [33] \\
\hline 20 & & & $\mathrm{~K}_{5} \mathrm{CoW}_{12} \mathrm{O}_{40} \cdot 3 \mathrm{H}_{2} \mathrm{O}$ & DCE, rt, 10-24 h & $83-92$ & [33] \\
\hline 21 & \multirow{2}{*}{$\begin{array}{l}\mathrm{Ph}, 4-\mathrm{BrPh}, 2-\mathrm{CIPh}, 4-\mathrm{CIPh}, 4-\mathrm{FPh} \\
\text { 4-CNPh, 3-OMePh, 3- } \mathrm{NO}_{2} \mathrm{Ph} \text { 4-MePh }\end{array}$} & \multirow{2}{*}{$\mathrm{Me}, \mathrm{Ph}, \mathrm{NH}_{2}$} & $\mathrm{HClO}_{4}-\mathrm{SiO}_{2}$ & DCE, $125^{\circ} \mathrm{C}, 6-8 \mathrm{~h}$ & $85-92$ & [34] \\
\hline 22 & & & $\mathrm{HClO}_{4}-\mathrm{SiO}_{2}$ & $125^{\circ} \mathrm{C}, 8-14 \min$ & $90-96$ & [34] \\
\hline 23 & \multirow{3}{*}{$\begin{array}{l}\mathrm{Ph}, 4-\mathrm{BrPh}, 4-\mathrm{ClPh}, 4-\mathrm{FPh}, 4-\mathrm{MePh} \\
\text { 4-NMe }{ }_{2} \mathrm{Ph}, 4-\mathrm{NO}_{2} \mathrm{Ph}, 4-\mathrm{OMePh} \\
\text { 3- } \mathrm{NO}_{2} \mathrm{Ph}, 3-\mathrm{FPh}, 3-\mathrm{OMePh}, 2,4-\mathrm{Cl}_{2} \mathrm{Ph} \text {, } \\
\text { 2,5-(OMe })_{2} \mathrm{Ph}, 3,4-(\mathrm{OMe})_{2} \mathrm{Ph}, 2-\mathrm{ClPh} \text {, } \\
\text { 2- } \mathrm{NO}_{2} \mathrm{Ph}, 2-\mathrm{MePh}\end{array}$} & \multirow{3}{*}{$\mathrm{Me}, \mathrm{Ph}$} & $\mathrm{HClO}_{4}-\mathrm{SiO}_{2}$ & $\mathrm{ACN}, 85^{\circ} \mathrm{C}, 20 \mathrm{~h}$ & $60-88$ & [35] \\
\hline 24 & & & $\mathrm{HClO}_{4}-\mathrm{SiO}_{2}$ & $110^{\circ} \mathrm{C}, 30-80 \mathrm{~min}$ & $76-91$ & [35] \\
\hline 25 & & & $\mathrm{HClO}_{4}-\mathrm{SiO}_{2}$ & $\begin{array}{l}\text { MW } 450 \mathrm{~W} \\
12-20 \mathrm{~min}\end{array}$ & $75-94$ & [35] \\
\hline 26 & $\begin{array}{l}\mathrm{Ph}, 4-\mathrm{ClPh}, 4-\mathrm{FPh}, 3-\mathrm{CF}_{3} \mathrm{Ph}, 3-\mathrm{NO}_{2} \mathrm{Ph} \text {, } \\
\text { 4-MePh, 4-EtPh, 4-OHPh, 4-MeOPh, } \\
\text { 3-OMe-4-OHPh, Et, iPr }\end{array}$ & $\begin{array}{l}\text { Me, } \mathrm{Ph}, \mathrm{NH}_{2} \text {, } \\
\text { vinyl }\end{array}$ & $\mathrm{HClO}_{4}-\mathrm{SiO}_{2}$ & $125^{\circ} \mathrm{C}, 5-9 \mathrm{~h}$ & $68-93$ & {$[36]$} \\
\hline 27 & $\begin{array}{l}\mathrm{Et} \text {, iPr, } \mathrm{CH}=\mathrm{CHPh}, \mathrm{C}_{5} \mathrm{H}_{10} \text {, cyclohexyl, } \\
\text { 2-pyridyl, } \mathrm{Ph}, 4-\mathrm{ClPh}, 3-\mathrm{OMePh} \text {, } \\
\text { 4-pyridyl }\end{array}$ & $\mathrm{Me}, \mathrm{Bn}$ & SSA & $\mathrm{rt}, 1.5-2.5 \mathrm{~h}$ & $79-85$ & {$[37]$} \\
\hline
\end{tabular}


Table 1: Comparison of various conditions applied in the synthesis of amidoalkylnaphthols 4 . (continued)

$\mathrm{Ph}, 4-\mathrm{CIPh}, 2-\mathrm{MePh}, 2-\mathrm{CIPh}, 3-\mathrm{NO}_{2} \mathrm{Ph}$,

28 4-FPh, 4-MePh, 4-BrPh, 2-OMePh, 3-OMePh

$29 \mathrm{Ph}, 4-\mathrm{MePh}, 4-\mathrm{NO}_{2} \mathrm{Ph}, 4-\mathrm{NMe}_{2} \mathrm{Ph}$,

30 4-CIPh, 4-BrPh, 4-OMePh,

3- $\mathrm{NO}_{2} \mathrm{Ph} 3-\mathrm{FPh}, 4-\mathrm{FPh}, 2,4-\mathrm{Cl}_{2} \mathrm{Ph}$,

2,5-(OMe) ${ }_{2} \mathrm{Ph}, 2-\mathrm{CIPh}, 3-\mathrm{OMePh}$,

$2-\mathrm{NO}_{2} \mathrm{Ph}, 2-\mathrm{MePh}, 3,4-(\mathrm{OMe})_{2} \mathrm{Ph}$

$32 \mathrm{Ph}, 4-\mathrm{NMe}_{2} \mathrm{Ph}, 4-\mathrm{OMePh}, 4-\mathrm{CIPh}$,

33 4-BrPh, 3- $\mathrm{NO}_{2} \mathrm{Ph}, 4-\mathrm{FPh}, 2,4-\mathrm{Cl}_{2} \mathrm{Ph}$,

2-CIPh, 3-OMePh, 2- $\mathrm{NO}_{2} \mathrm{Ph}, 3-\mathrm{OMePh}, \quad \mathrm{Me}, \mathrm{Ph}$

2- $\mathrm{NO}_{2} \mathrm{Ph}, 2-\mathrm{MePh}, 3,4-(\mathrm{OMe})_{2} \mathrm{Ph}$,

34 4-MePh, 4- $\mathrm{NO}_{2} \mathrm{Ph}, 3-\mathrm{FPh}$,

2,5-(OMe $)_{2} \mathrm{Ph}$

35

$\mathrm{Ph}, 4-\mathrm{OMePh}, 4-\mathrm{MePh}, 4-\mathrm{CIPh}$,

3- $\mathrm{NO}_{2} \mathrm{Ph}, 2$-furyl

$36 \mathrm{Ph}, 4-\mathrm{OMePh}, 4-\mathrm{MePh}, 4-\mathrm{CIPh}$

$4-\mathrm{NO}_{2} \mathrm{Ph}, 3-\mathrm{NO}_{2} \mathrm{Ph}, 2,4-\mathrm{Cl}_{2} \mathrm{Ph}, 2-\mathrm{ClPh}, \quad \mathrm{Me}, \mathrm{Ph}$

37

2-MePh

$38 \mathrm{Ph}, 4-\mathrm{ClPh}, 4-\mathrm{BrPh}, 4-\mathrm{FPh}, 4-\mathrm{CNPh}$,

3-NO ${ }_{2} \mathrm{Ph}, 3-\mathrm{OMePh}, 2-\mathrm{CIPh}$

$\mathrm{Me}$

Me

$\mathrm{ClSO}_{3} \mathrm{H}$

$\mathrm{NaHSO}_{4} \cdot \mathrm{H}_{2} \mathrm{O}$

$\mathrm{NaHSO}_{4} \cdot \mathrm{H}_{2} \mathrm{O}$

$\mathrm{NaHSO}_{4} \cdot \mathrm{H}_{2} \mathrm{O}$

$\mathrm{Fe}\left(\mathrm{HSO}_{4}\right)_{3}$

$\mathrm{Fe}\left(\mathrm{HSO}_{4}\right)_{3}$

$\mathrm{Fe}\left(\mathrm{HSO}_{4}\right)_{3}$

$39 \mathrm{Ph}, 3-\mathrm{NO}_{2} \mathrm{Ph}, 4-\mathrm{CIPh}, 2-\mathrm{MePh}, 2-\mathrm{CIPh}$, 4-FPh, 3- $\mathrm{NO}_{2} \mathrm{Ph}, 4-\mathrm{BrPh}, 3-\mathrm{BrPh}$,

40 2-BrPh, 2-furyl

$\mathrm{Ph}$, 4-CIPh, 4-OMePh, 3- $\mathrm{NO}_{2} \mathrm{Ph}$, 2-furyl, 2-CIPh, Et

$\mathrm{Ph}, 4-\mathrm{NO}_{2} \mathrm{Ph}, 3-\mathrm{NO}_{2} \mathrm{Ph}, 2-\mathrm{NO}_{2} \mathrm{Ph}$,

42 4-CIPh, 2-CIPh, 4-OMePh, 2-OMePh,

4-MePh, 2,4- $\mathrm{Cl}_{2} \mathrm{Ph}, 4-\mathrm{NMe}_{2} \mathrm{Ph}$

$\mathrm{Ph}, 4-\mathrm{NO}_{2} \mathrm{Ph}, 3-\mathrm{NO}_{2} \mathrm{Ph}, 2-\mathrm{NO}_{2} \mathrm{Ph}$

4-CN-Ph, 4-FPh, 3-FPh, 4-BrPh, 2-CIPh,

43 2,4- $\mathrm{Cl}_{2} \mathrm{Ph}, 4-\mathrm{CIPh}, 2-\mathrm{MePh}, 4-\mathrm{MePh}$,

3-OMePh, 4-OMePh, 3,4-(OMe) ${ }_{2} \mathrm{Ph}$,

$\mathrm{CH}=\mathrm{CH}-\mathrm{Ph}$

$\mathrm{Ph}$, 4-OHPh, 4-CIPh, 2-CIPh, 4- $\mathrm{NO}_{2} \mathrm{Ph}$,

44 3- $\mathrm{NO}_{2} \mathrm{Ph}, 4-\mathrm{OMePh}$, Et, 4-NMe ${ }_{2} \mathrm{Ph}$,

$3,4,5-(\mathrm{OMe})_{3} \mathrm{Ph}$

$\mathrm{Ph}, 3,4,5,-(\mathrm{OMe})_{3} \mathrm{Ph}, 4-\mathrm{OMePh}$,

2,3- $\mathrm{Me}_{2} \mathrm{Ph}, 4-\mathrm{FPh}, 4-\mathrm{CIPh}, 2-\mathrm{OHPh}$,

45 4- $\mathrm{NO}_{2} \mathrm{Ph}, 2,4-\mathrm{Cl}_{2} \mathrm{Ph}, 2-\mathrm{OMePh}, 2-\mathrm{CIPh}, \quad \mathrm{Me}, \mathrm{Ph}$

2-BrPh, 3-BrPh, 3-FPh, 3-CIPh

4-(CHO)Ph, Et, 4-CNPh, 4-IPh

$\mathrm{Ph}, 4-\mathrm{CIPh}, 4-\mathrm{OMePh}, 4-\mathrm{MePh}$,

4-NMe ${ }_{2} \mathrm{Ph}, 4-\mathrm{NO}_{2} \mathrm{Ph}, 2-\mathrm{NO}_{2} \mathrm{Ph}, 2-\mathrm{CIPh}$,

46 2,4- $\mathrm{Cl}_{2} \mathrm{Ph}, 4-\mathrm{OH}-3-\mathrm{OMePh}, 3-\mathrm{OMePh}$,

$3-\mathrm{NO}_{2} \mathrm{Ph}, 4-\mathrm{FPh}, 2,5-(\mathrm{OMe})_{2} \mathrm{Ph}$,

3,4-(OMe) ${ }_{2} \mathrm{Ph}, 2-\mathrm{MePh}, 4-\mathrm{OHPh}, 3-\mathrm{CIPh}$

47

$48 \mathrm{Ph}, 4-\mathrm{CIPh}, 4-\mathrm{NMe}_{2} \mathrm{Ph}, 3-\mathrm{NO}_{2} \mathrm{Ph}$, 2,5-(OMe $)_{2}$

49

50

$51 \mathrm{Ph}, 4-\mathrm{CIPh}, 4-\mathrm{NMe}_{2} \mathrm{Ph}, 4-\mathrm{MePh}$,

$3-\mathrm{NO}_{2} \mathrm{Ph}, 2,5-(\mathrm{OMe})_{2} \mathrm{Ph}$

52
$\mathrm{Me}, \mathrm{Ph}, \mathrm{NH}_{2}$,

vinyl,

2-thiophenyl

$\mathrm{Me}, \mathrm{Ph}, \mathrm{NH}_{2}$

Me

$\mathrm{Me}, \mathrm{Ph}, \mathrm{NH}_{2}$

Sr(

$\mathrm{Sr}(\mathrm{OTf})_{2} \quad \mathrm{CHCl}_{3}, 60^{\circ} \mathrm{C}, 8-15 \mathrm{~h} \quad 80-96$

CuPW

CuPMo

wet-TCT

sulfamic acid

sulfamic acid

$\mathrm{I}_{2}$

$\mathrm{P}_{2} \mathrm{O}_{5}$

$\mathrm{Bu}_{4} \mathrm{NBr}, 100^{\circ} \mathrm{C}$,

90 min 74-95

$\mathrm{Bu}_{4} \mathrm{NBr}, 100^{\circ} \mathrm{C}$,

70-93

$100{ }^{\circ} \mathrm{C}, 8-14 \mathrm{~min} \quad 90-96$

[43]

sonication, $28-30{ }^{\circ} \mathrm{C}$,

10-60 min

55-92

[44]

sonication, DCE,

28-30 ${ }^{\circ} \mathrm{C}, 25-120 \mathrm{~min}$ 78-94

[44]

DCE, rt, 8-24 h 30-93

$\mathrm{Me}, \mathrm{Ph}$

$60{ }^{\circ} \mathrm{C}, 5-15 \mathrm{~min}$

80-97

[46]

$\mathrm{Me}, \mathrm{Ph}$

$\mathrm{P}_{2} \mathrm{O}_{5} \cdot \mathrm{SiO}_{2}$

$100{ }^{\circ} \mathrm{C}, 3-40 \mathrm{~min}$

54-94

[47]

TBBDA

rt, 30-80 min

88-97

[48]

MSI

[Bpy]BF ${ }_{4}, 80^{\circ} \mathrm{C}$, 25-60 min

82-95

$\mathrm{Me}, \mathrm{Ph}, \mathrm{NH}_{2} \quad$ succinic acid

$120^{\circ} \mathrm{C}, 3-60 \mathrm{~min}$

65-98

tannic acid

MW $480 \mathrm{~W}$, 5-13 $\min$ 85-90

[51]

tannic acid

oil bath, $110-120^{\circ} \mathrm{C}$,

7-20 min

75-90

tannic acid

hot plate, $110-120^{\circ} \mathrm{C}$

10-21 $\mathrm{min}$

47-76

[51]

p-nitrobenzoic acid

MW $450 \mathrm{~W}, 8-14 \mathrm{~min} \quad$ 82-92

p-nitrobenzoic acid

oil bath, $110-120^{\circ} \mathrm{C}$,

80-90

[52]

p-nitrobenzoic acid

12-26 min

$60-74$

[52] 
Table 1: Comparison of various conditions applied in the synthesis of amidoalkylnaphthols 4 . (continued)

\begin{tabular}{|c|c|c|c|c|c|c|}
\hline 53 & $\begin{array}{l}\mathrm{Ph}, 4-\mathrm{BrPh}, 2-\mathrm{CIPh}, 4-\mathrm{CIPh}, 2,4-\mathrm{Cl}_{2} \mathrm{Ph} \\
\text { 4-FPh, 4-OMePh, 4-MePh, 3- } \mathrm{NO}_{2} \mathrm{Ph} \\
\text { 4- } \mathrm{NO}_{2} \mathrm{Ph}\end{array}$ & $\mathrm{Me}$ & CBSA & $130^{\circ} \mathrm{C}, 2-20 \mathrm{~min}$ & $86-93$ & {$[53]$} \\
\hline 54 & $\begin{array}{l}\mathrm{Ph}, 4-\mathrm{ClPh}, 4-\mathrm{OMe}, 4-\mathrm{NO}_{2} \mathrm{Ph}, 2-\mathrm{NO}_{2} \mathrm{Ph} \\
\text { 2-CIPh, 4-MePh }\end{array}$ & $\mathrm{Me}, \mathrm{Ph}, \mathrm{NH}_{2}$ & citric acid & $120^{\circ} \mathrm{C}, 7-43 \mathrm{~min}$ & $87-94$ & [54] \\
\hline 55 & & & sulfanilic acid & MW $450 \mathrm{~W}, 8-14 \mathrm{~min}$ & $83-94$ & [55] \\
\hline 56 & $\begin{array}{l}\mathrm{Ph}, 4-\mathrm{ClPh}, 4-\mathrm{NMe}_{2} \mathrm{Ph}, 4-\mathrm{MePh} \text {, } \\
\text { 3- } \mathrm{NO}_{2} \mathrm{Ph}, 2,5-(\mathrm{OMe})_{2} \mathrm{Ph} \text {, 2-thiophene, }\end{array}$ & $\mathrm{Me}, \mathrm{Ph}, \mathrm{NH}_{2}$ & sulfanilic acid & $\begin{array}{l}\text { oil bath, } 110-120^{\circ} \mathrm{C} \text {, } \\
12-24 \min \end{array}$ & $80-95$ & [55] \\
\hline 57 & 1-Nph, 2-Cl-5-FPh & & sulfanilic acid & $\begin{array}{l}\text { hot plate, } 110-115^{\circ} \mathrm{C} \text {, } \\
11-28 \mathrm{~min}\end{array}$ & $62-72$ & [55] \\
\hline 58 & $\begin{array}{l}\mathrm{Ph}, 2-\mathrm{NO}_{2} \mathrm{Ph}, 3-\mathrm{NO}_{2} \mathrm{Ph}, 4-\mathrm{NO}_{2} \mathrm{Ph} \text {, } \\
\text { 2-ClPh, 4-CIPh, 2,4-Cl } \mathrm{Ph}, 4-\mathrm{MePh} \text {, } \\
\text { 4-MeOPh, Et, Pr }\end{array}$ & $\mathrm{Me}, \mathrm{Ph}, \mathrm{NH}_{2}$ & $\mathrm{Bi}\left(\mathrm{NO}_{3}\right)_{3} \cdot 5 \mathrm{H}_{2} \mathrm{O}$ & $80^{\circ} \mathrm{C}, 6-150 \mathrm{~min}$ & $79-97$ & {$[56]$} \\
\hline 59 & $\mathrm{Ph}, 4-\mathrm{ClPh}, 4-\mathrm{BrPh}, 3-\mathrm{NO}_{2} \mathrm{Ph}, 4-\mathrm{FPh}, \mathrm{Et}$ & $\mathrm{Me}, \mathrm{Ph}, \mathrm{NH}_{2}$ & $\begin{array}{l}\text { 1-hexanesulfonic } \\
\text { acid sodium salt }\end{array}$ & MW, 3-20 min & $35-95$ & [57] \\
\hline 60 & $\begin{array}{l}\mathrm{Ph}, 4-\mathrm{NO}_{2} \mathrm{Ph}, 3-\mathrm{NO}_{2} \mathrm{Ph}, 4-\mathrm{OMePh} \\
\text { 4-iPrPh, 2-BrPh, } \mathrm{CH}_{2}-\mathrm{CH}_{2}-\mathrm{Ph} \text {, } \\
\mathrm{CH}=\mathrm{CH}-\mathrm{Ph}, \mathrm{C}_{11} \mathrm{H}_{23}, 9-\text { phenanthrenyl, } \\
\text { 1-pyrenyl }\end{array}$ & $\mathrm{Me}, \mathrm{Ph}$ & $\mathrm{ZrO}(\mathrm{OTf})_{2}$ & $80^{\circ} \mathrm{C}, 1.5-10 \mathrm{~min}$ & $65-98$ & [58] \\
\hline 61 & $\begin{array}{l}\mathrm{Ph}, 3-\mathrm{NO}_{2} \mathrm{Ph}, 4-\mathrm{NO}_{2} \mathrm{Ph}, 3-\mathrm{FPh}, 4-\mathrm{FPh} \\
\text { 4-OMePh, 2-OMePh }\end{array}$ & $\mathrm{Me}, \mathrm{Ph}$ & $\mathrm{SO}_{3} \mathrm{H}$-carbon & $100^{\circ} \mathrm{C}, 30 \mathrm{~min}$ & $71-96$ & [59] \\
\hline 62 & $\begin{array}{l}\mathrm{Ph}, 4-\mathrm{ClPh}, 4-\mathrm{BrPh}, 4-\mathrm{EPh}, 4-\mathrm{MePh} \\
\text { 4-OHPh, 3-OHPh, 4-OMePh, 4-OEtPh, } \\
\text { 3- } \mathrm{NO}_{2} \mathrm{Ph}, 2-\mathrm{NO}_{2} \mathrm{Ph}, \mathrm{iPr}\end{array}$ & $\mathrm{Me}$ & $\begin{array}{l}\text { MCM-41-N-propyl- } \\
\text { sulfamic acid }\end{array}$ & $130^{\circ} \mathrm{C}, 90-270 \mathrm{~min}$ & $35-98$ & {$[60]$} \\
\hline 63 & $\begin{array}{l}\mathrm{Ph}, 2-\mathrm{ClPh}, 4-\mathrm{MePh}, 3-\mathrm{NO}_{2} \mathrm{Ph} \\
\text { 2-NO } \\
2 \mathrm{Ph}, 4-\mathrm{OMePh}, 4-\mathrm{BrPh}, 4-\mathrm{CIPh}\end{array}$ & $\begin{array}{l}\mathrm{Me}, \mathrm{Ph}, \mathrm{NH}_{2} \\
\mathrm{NHMe} \text {, vinyl }\end{array}$ & $\begin{array}{l}\text { polyphosphate } \\
\text { ester }\end{array}$ & $80^{\circ} \mathrm{C}, 10-20 \mathrm{~min}$ & $85-93$ & [61] \\
\hline 64 & $\begin{array}{l}\mathrm{Ph}, 4-\mathrm{MePh}, 2-\mathrm{MePh}, 4-\mathrm{OMePh} \\
\text { 3-OMePh, 3,4-(OMe) })_{2} \mathrm{Ph}, 4-\mathrm{NMe}_{2} \mathrm{Ph} \\
\text { 4- } \mathrm{NO}_{2} \mathrm{Ph}, 3-\mathrm{NO}_{2} \mathrm{Ph}, 2-\mathrm{NO}_{2} \mathrm{Ph}, 4-\mathrm{CIPh} \text {, } \\
\text { 2-CIPh, 4-BrPh, 4-FPh, 2,4-Cl }{ }_{2} \mathrm{Ph}\end{array}$ & $\mathrm{Me}$ & Amberlite IR-120 & MW $360 \mathrm{~W}, 3-6 \mathrm{~min}$ & $91-96$ & [62] \\
\hline
\end{tabular}

sonication for 10-30 minutes led to improved yields of $90-98 \%$.

There are previous examples for the synthesis of 1-amidoalkyl2-naphthols carried out in the presence of Lewis and Brønsted acid catalysts. As depicted in Table 1, entries 13-38, the applicability of $p$-toluenesulfonic acid ( $p$-TSA) [27], montmorillonite $\mathrm{K} 10$ [30], Indion-130 [31], iodine $\left(\mathrm{I}_{2}\right)$ [32], potassium dodecatungstocobaltate $\left(\mathrm{K}_{5} \mathrm{CoW}_{12} \mathrm{O}_{40} \cdot 3 \mathrm{H}_{2} \mathrm{O}\right)$ [33], silica-supported perchloric acid $\left(\mathrm{HClO}_{4}-\mathrm{SiO}_{2}\right)$ [34-36] and sulfuric acid [35], chlorosulfonic acid [38], sodium hydrogen sulfate $\left(\mathrm{NaHSO}_{4} \cdot \mathrm{H}_{2} \mathrm{O}\right)$ [39], ferric(III) hydrogen sulfate $\left[\mathrm{Fe}\left(\mathrm{HSO}_{4}\right)_{3}\right.$; 40], strontium(II) triflate, $\mathrm{Sr}(\mathrm{OTf})_{2} ;$ [41], copper-exchanged heteropoly acids, $\mathrm{Cu}_{1 \cdot 5} \mathrm{PMo}_{12} \mathrm{O}_{40}(\mathrm{CuPMo})$ and $\mathrm{Cu}_{1 \cdot 5} \mathrm{PW}_{12} \mathrm{O}_{40}$ (CuPW); [42] or wet cyanuric acid (wet-TCT) [43] was also tested. These methods suffer from a number of drawbacks, such as strong acidic media, high temperature, and prolonged reactions. Furthermore, the yields are often not satisfactory.

To eliminate the disadvantages of previous strategies, Samant et al. reported an ultrasound-promoted condensation catalysed by sulfamic acid [44]. As shown in Table 1, entries 39 and 40, both dichloroethane (DCE) and solvent-free conditions were tested. The catalyst worked at low temperature $\left(28-30^{\circ} \mathrm{C}\right)$ and the products were formed in short reaction times in up to $94 \%$ yields. Shinde et al. also published iodine catalysis carried out at room temperature in DCE [45]. Whereas long reaction times were needed in the latter process, good yields could be achieved under mild conditions.

In additional publications listed in Table 1, entries 42-64, phosphorus pentoxide $\left(\mathrm{P}_{2} \mathrm{O}_{5}\right)$ [46], silica-supported phosphorus pentoxide $\left(\mathrm{P}_{2} \mathrm{O}_{5}-\mathrm{SiO}_{2}\right)$ [47], $N, N, N^{\prime}, N^{\prime}$-tetrabromobenzene-1,3disulfonamide (TBBDA) [48], 1-methyl-3-(2-(sulfoxy)ethyl)$1 \mathrm{H}$-imidazol-3-ium chloride (MSI) [49], succinic acid [50], tannic acid [49], p-nitrobenzoic acid [52], a carbon-based solid acid (CBSA) [53], citric acid [54], sulfanilic acid [55], bismuth(III) nitrate pentahydrate $\left(\mathrm{Bi}\left(\mathrm{NO}_{3}\right)_{3} \cdot 5 \mathrm{H}_{2} \mathrm{O}\right)$ [56], 1-hexanesulfonic acid sodium salt [57], zirconyl triflate $\left(\mathrm{ZrO}(\mathrm{OTf})_{2}\right)[58]$, sulfonated carbon ( $\mathrm{SO}_{3} \mathrm{H}$-carbon) [59], MCM-41- $N$-propylsulfamic acid [60], polyphosphate ester [61] and amberlite IR-120 [62] were used as catalysts. These latest strategies provide efficient syntheses under mild conditions without using harsh chemicals. Furthermore, the application of 
microwave irradiation or sonication is also preferred to conventional heating methods to accelerate the reactions.

\section{Synthesis of aminoalkylphenols}

The mechanism of the formation of phenolic Mannich bases is similar to that discussed above for the synthesis of amidoalkylnaphthols. First, the phenol component reacts with the aldehyde to form the $o$-QM intermediate, which reacts in a nucleophilic addition step with the amine component, resulting in aminoalkylphenol derivatives. A few examples are summarized in Table 2. An important difference although must be noted. In the case of aminonalkylnaphthols, the $o$-QM intermediate partially remains aromatic while the formation of phenolic $o$-QMs leads to the loss of the aromaticity of the only aromatic ring present. This results in differences in both the formation and stability of $o-\mathrm{QM}$.

Grimaud et al. synthesized Mannich bases starting from $\mathrm{N}$-benzylpiperazine, various aldehydes and substituted phenols [63]. The intermediate Mannich adducts $8 \mathbf{8}-\mathbf{i}$ were then reacted with 5,5-dimethylcyclohexane-1,3-dione or 1-methyl- $1 H$-indole in the presence of lithium perchlorate as catalyst to afford the new 3,3-dimethyl-2,3,4,9-tetrahydro- $1 H$-xanthen-1-ones and 3 -substituted indoles. The process was then extended to isocyanides and new aminobenzofurans formed via $[4+1]$ cycloaddition were isolated.

Bharate et al. reported ortho-amidoalkylation of phenols in which a tandem Knoevenagel condensation occurs through
$o$-QM followed by the formation of an unstable oxazine intermediate [64]. Later, the same research group published a similar reaction extended by various lactams carried out in trifluoroacetic acid in water [65]. As reported in both papers, Mannich bases formed 9a-t were isolated in good yields. Plausible reaction pathways were described and the energetic values of the transition states were calculated.

In one of the latest publications with respect to this topic, Priya et al. disclosed the synthesis of a wide range of novel 2-[(benzo[ $d]$ thiazol-2-ylamino(phenyl)methyl]phenols 10a-m [66]. In their study, 2-amino-1,3-benzothiazoles, various aldehydes and substituted phenols were reacted in the presence of $\mathrm{ZnCl}_{2}$ as catalyst.

\section{Reactions of o-QMs formed by Mannich bases}

As the formation of Mannich bases can be explained by the generation of an $o$-QM intermediate followed by the nucleophilic addition of the amine component, the reverse reaction with the corresponding nucleophile is also feasible. Mechanistically, the Mannich adduct generates an $o-\mathrm{QM}$ via the loss of an amine, then this reactive intermediate reacts with the nucleophile (dienophile) species in different reactions to form a wide range of heterocyclic compounds.

\section{Reactions with $\mathrm{C}=\mathrm{C}$ dienophiles}

Reactions of $o$-QMs with different $\mathrm{C}=\mathrm{C}$ dienophiles are listed in Table 3. Osyanin et al. reported the efficient reaction of quater-

Table 2: Formation and substrate scope of phenolic Mannich bases.

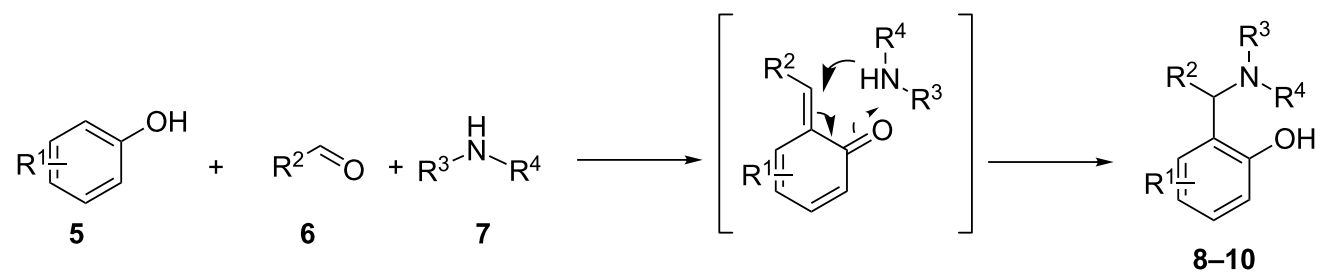

\begin{tabular}{|c|c|c|c|c|c|}
\hline entry & $\mathrm{R}^{1}$ & $\mathrm{R}^{2}$ & $\mathrm{R}^{3}-\mathrm{H}-\mathrm{R}^{4}$ & product & reference \\
\hline 1 & 4-Me, 4-COOMe & $\begin{array}{c}\mathrm{Ph}, 4-\mathrm{MeOPh}, \\
\text { 4- } \mathrm{NO}_{2} \mathrm{Ph}, 3-\mathrm{CF}_{3} \mathrm{Ph} \text {, } \\
\text { 2-All-O-Ph }\end{array}$ & & $8 a-i$ & [63] \\
\hline 2 & $\begin{array}{c}\mathrm{H}, 2-\mathrm{Me}, 3,5-(\mathrm{OH})_{2}-2,4-(\mathrm{CHO})_{2} \\
\text { 3,5-(OH)})_{2}-2,4-\mathrm{Ac}_{2} \\
\text { 3,5-(OH })_{2}-2,4-\text { dipropanoyl, } \\
\text { 3,5-(OH })_{2}-2,4-\text { diisobutanoyl, } \\
\text { 3,5-(OH })_{2}-2,4 \text {-dibutanoyl }\end{array}$ & $\mathrm{H}$ & 0 & $9 a-t$ & {$[64,65]$} \\
\hline 3 & $\begin{array}{c}\text { 3-Cl, 2- } \mathrm{NO}_{2}, 4-\mathrm{OMe}, 4-\mathrm{NO}_{2} \\
4-\mathrm{Cl}, 2,4-\mathrm{Cl}_{2}\end{array}$ & $\begin{array}{c}\text { 4-BrPh, 4- } \mathrm{NO}_{2} \mathrm{Ph} \\
\text { 4-CIPh, 4-OMePh, } \\
\text { 2,3- } \mathrm{Me}_{2} \mathrm{Ph}, 4-t-\mathrm{BuPh}\end{array}$ & & $10 a-m$ & {$[66]$} \\
\hline
\end{tabular}


Table 3: Reactions of o-QMs with different dienophile species.

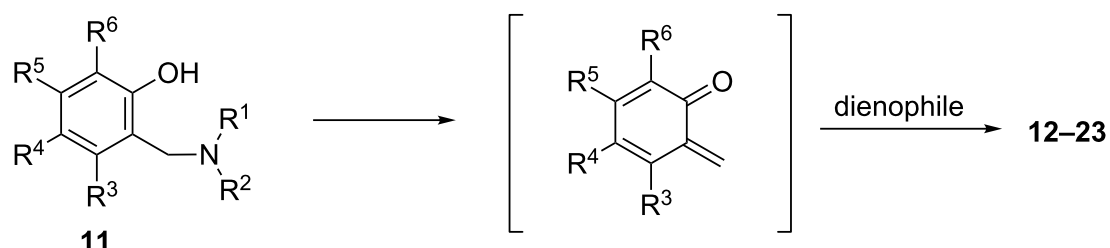

$\left.\right|_{R^{1}-N-R^{2}} R^{3} \quad R^{4} \quad R^{5} \quad R^{6} \quad$ dienophile

$\mathrm{NMe}_{3}{ }^{+} \mathrm{H} \quad \begin{gathered}\mathrm{Me}, \mathrm{Ad}, t-\mathrm{Bu}, \\ \mathrm{Ac}, \mathrm{Bn}, \mathrm{Cl}\end{gathered} \quad \mathrm{H} \quad \mathrm{H} \quad \mathrm{CH}_{2}(\mathrm{CN})_{2}$<smiles>[R4]c1ccc2c(c1)CC(C#N)=C(N)O2</smiles>

12

$\mathrm{CH}_{2}(\mathrm{CN})_{2}$<smiles>Cc1cc(C#N)c2c(c1)CC(C#N)=C(N)O2</smiles>

13<smiles>C#CC(C#N)c1ccc2c(c1)OC(N)=C(C#N)C2</smiles>

14<smiles>Cc1cc2c(cc1C)OC(N)=C(C#N)C2</smiles>

15<smiles></smiles>

$[68]$

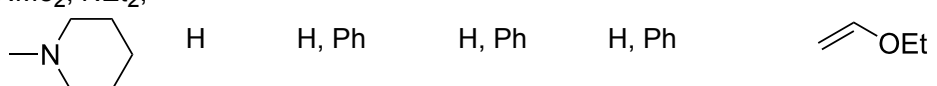

$-\mathrm{O} O \mathrm{OH} \quad \mathrm{H} \quad \mathrm{H}$ COMe<smiles>CC(=O)c1ccc2c(c1)CC1(C)CCO[C@]1(C)O2</smiles>

[69]

17<smiles>CC(=O)c1ccc2c(c1)C[C@H]1[C@@H](C)CO[C@@]1(C)O2</smiles>

[69]

18<smiles>COc1cc2c(c(OC)c1C(C)C)OC1=C(C2)C(=O)CC(C)(C)C1</smiles>

[70]

19<smiles>[R4]c1cc([R6])c2c(c1)CC1=C(CC(C)(C)CC1=O)O2</smiles>

20 
Table 3: Reactions of o-QMs with different dienophile species. (continued)

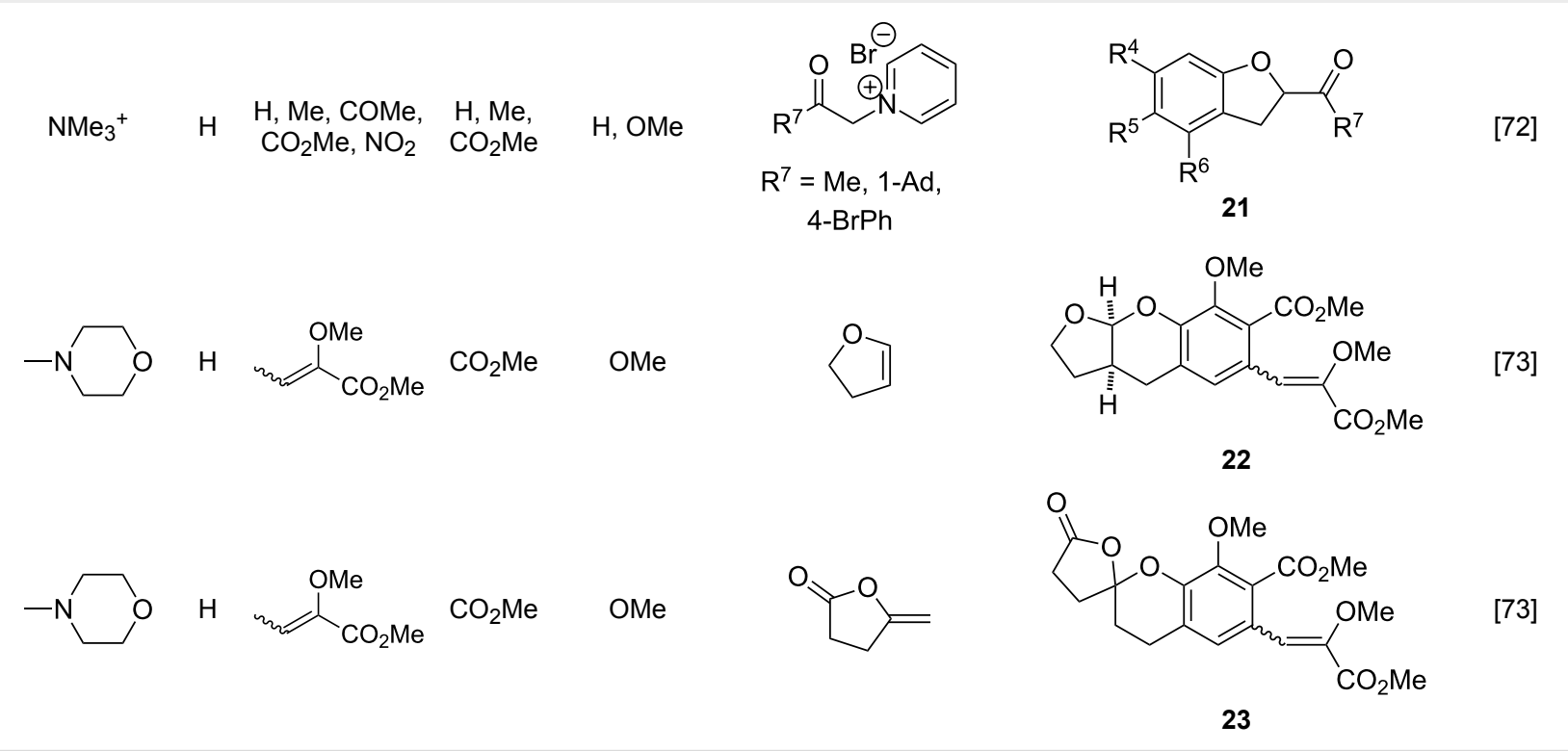

nary ammonium salt Mannich bases with malononitrile catalysed by 1,8-diazabicyclo[5.4.0]undec-7-ene (DBU) [67]. It is known that the use of quaternary ammonium salts offers the easier removal of the amino residue and, therefore, trapping the transient electrophilic species at lower temperature. Carrying out the reactions in protic solvents such as $\mathrm{H}_{2} \mathrm{O}$ or EtOH at $100{ }^{\circ} \mathrm{C}$, the desired products were formed in short reactions (1-20 $\mathrm{min}$ ) and chromene-2-carbonitriles 12-15 were isolated in $61-88 \%$ yields.

Saito et al. generated $o$-QMs starting from Mannich bases by low-energy UV irradiation in aqueous acetonitrile [68]. In the presence of a large excess of ethyl vinyl ether as $o$-QM trapping agent, they isolated several 2-ethoxychromane derivatives 16. However, yields as low as $36 \%$ were found in some cases.

$o$-QMs derived from Mannich adducts also appear to be key intermediates in the syntheses of biologically active natural compounds. Wilson et al. successfully achieved the total synthesis of xyloketals $\mathbf{1 7}$ and $\mathbf{1 8}$, including cycloaddition of substituted dihydrofurans and 1-(2,4-dihydroxy-3-(morpholinomethyl)phenyl)ethanone via $o-\mathrm{QM}$ intermediates [69]. Osyanin et al. reported the synthesis of Uvaria scheffleri alkaloids espintanol and $( \pm)$-schefflone starting from 6-((dibenzylamino)methyl)-3-isopropyl-2,4-dimethoxyphenol. The $o$-QM formed be trapped by 3-(dimethylamino)-5,5-dimethylcyclohex-2-en-1one resulting in tetrahydro- $1 H$-xanten-1-one 19 [70]. Starting from phenolic Mannich bases and 3-dimethylamino-2-cyclohexen-1-ones, the synthesis of 2,3,4,9-tetrahydro- $1 H$-xantene-1ones (20) has been published by the same research group.
The synthetic protocol was then extended to isolate benzo $[a]$ xanthen-11-ones or chromeno[3,2- $g] \beta$-carboline-8,13dione starting from 2 -naphthol and $1 H$ - $\beta$-carboline-1-one Mannich bases [71]. Although a high temperature was needed (reflux at $153{ }^{\circ} \mathrm{C}$ for 4 hours), the desired products were isolated in good (53-91\%) yields. The authors reported better results with the use of polyheterocyclic initial compounds. This can be explained by a dearomatisation step taking place in the transformation of phenolic Mannich bases, leading to the disappearance of the only aromatic ring. In a recent publication by same research group [72], they elaborated a simple route to 1,2-dihydronaphtho[2,1-b]furan and 2,3-dihydrobenzofurans via baseinduced desamination. They also reported the development of a simple, general route to 2,3-dihydrobenzofurans 21 starting from phenolic Mannich bases. The syntheses were also extended to 2-naphthol Mannich bases as initial compounds affording C-2-substituted 1,2-dihydronaphtho[2,1b]furans.

Bray et al. reacted ortho-hydroxybenzylamines with 2,3dihydrofuran and $\gamma$-methylene- $\gamma$-butyrolactone in DMF at $130{ }^{\circ} \mathrm{C}$ [73]. This method could successfully be applied in the synthesis of the spiroketal core of rubromycins 22 and $\mathbf{2 3}$.

One of the latest publications around the topic is published by Hayashi et al. in 2015 [74]. Starting from diarylmethylamines 24 and arylboroxines, they successfully developed a rhodiumcatalyzed asymmetric arylation process leading to triarylmethanes 25. With the application of mild reaction conditions ( $40{ }^{\circ} \mathrm{C}, 15 \mathrm{~h}$ ), a high enantioselectivity ( $\geq 90 \%$ ee) was reached with good to excellent yields. (Scheme 2 ). 


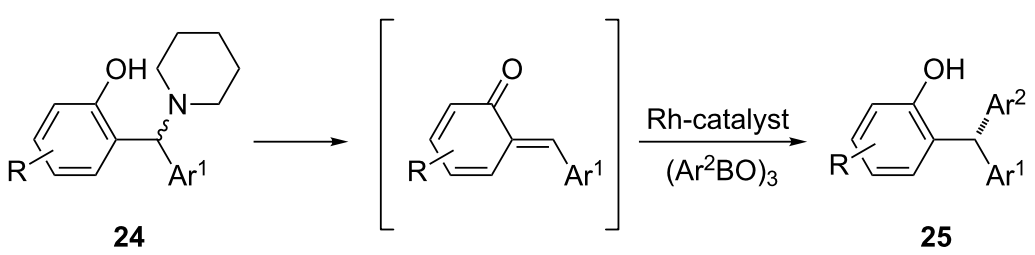

Scheme 2: Asymmetric syntheses of triarylmethanes starting from diarylmethylamines.

Starting from 2-naphthol, 2,2-disubstituted 3-hydroxypropanals and cyclic secondary amines, Jha et al. achieved the synthesis of 2,2-dialkyl-3-dialkylamino-2,3-dihydro- $1 H$-naphtho[2,1$b]$ pyrans under solvent-free conditions using $p$ TSA as catalyst [75]. It is important to note, that during the reaction, 2,2-disubstituted 3-hydroxypropanals $\mathbf{2 6}$ decompose to formaldehyde and 2,2-disubstituted acetaldehydes $\mathbf{2 8}$. Formaldehyde, as a nonenolizable compound is more likely to give Mannich base product 30. In contrast, enolizable 2,2-disubstituted aldehydes easily form enamines 31 that undergo cycloaddition with electrondeficient $o$-QMs giving 2,2-dialkyl-3-dialkylamino-2,3dihydro- $1 H$-naphtho[2,1-b]pyrans 32. A plausible mechanism is depicted in Scheme 3.

Watt et al. achieved the regioselective condensation of $\operatorname{bis}(N, N$ dimethylamino)methane with various hydroxyisoflavonoids to synthesize C-6- and C-8-substituted isoflavonoids $\mathbf{3 3}$ and $\mathbf{3 4}$ in a Mannich-type reaction [76]. These $o-\mathrm{QM}$ precursors by a thermal elimination of dimethylamine were then reacted with differ- ent cyclic dienophiles to give various inverse electron-demand Diels-Alder adducts 35-37. In case of 36, the cis-fused ring system found to be similar to bioactive xyloketals isolated from fungi (Scheme 4)

$o$-QMs are also known to undergo oligomerization in the absence of dienophiles and nucleophiles via an oxo-Diels-Alder protocol (Table 4). During the syntheses of 1,4,9,10anthradiquinones with potential antitumor activity, Kucklaender et al. isolated new spiro derivatives 38 [77]. These latter spirocyclic dimers formed in a Diels-Alder dimerization process by heating the corresponding Mannich bases under reflux in dichloromethane for 2 hours.

In the synthesis of 3,4-dihydro-2-aryl-2H-benzo[f]chromenes and 2-aryl-6,7-dimethylchromans starting from substituted styrenes and 1-dimethylaminomethyl-2-naphthol or 2-dimethylaminomethyl-4,5-dimethylphenol, Bilgiç et al. detected the formation of both dimers $\mathbf{3 9}$ and $\mathbf{4 0}$ of $o$-QMs formed by the ther-

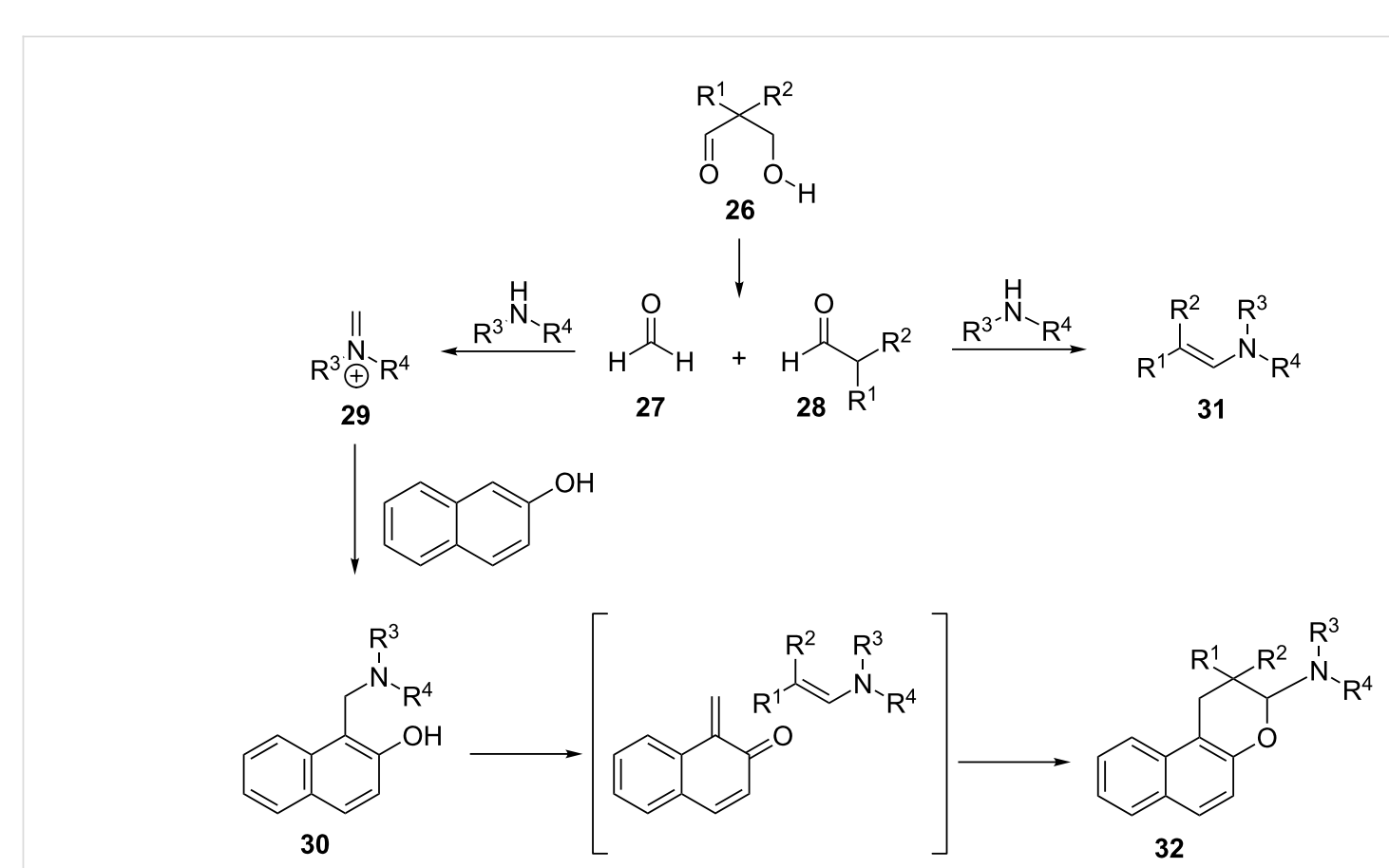




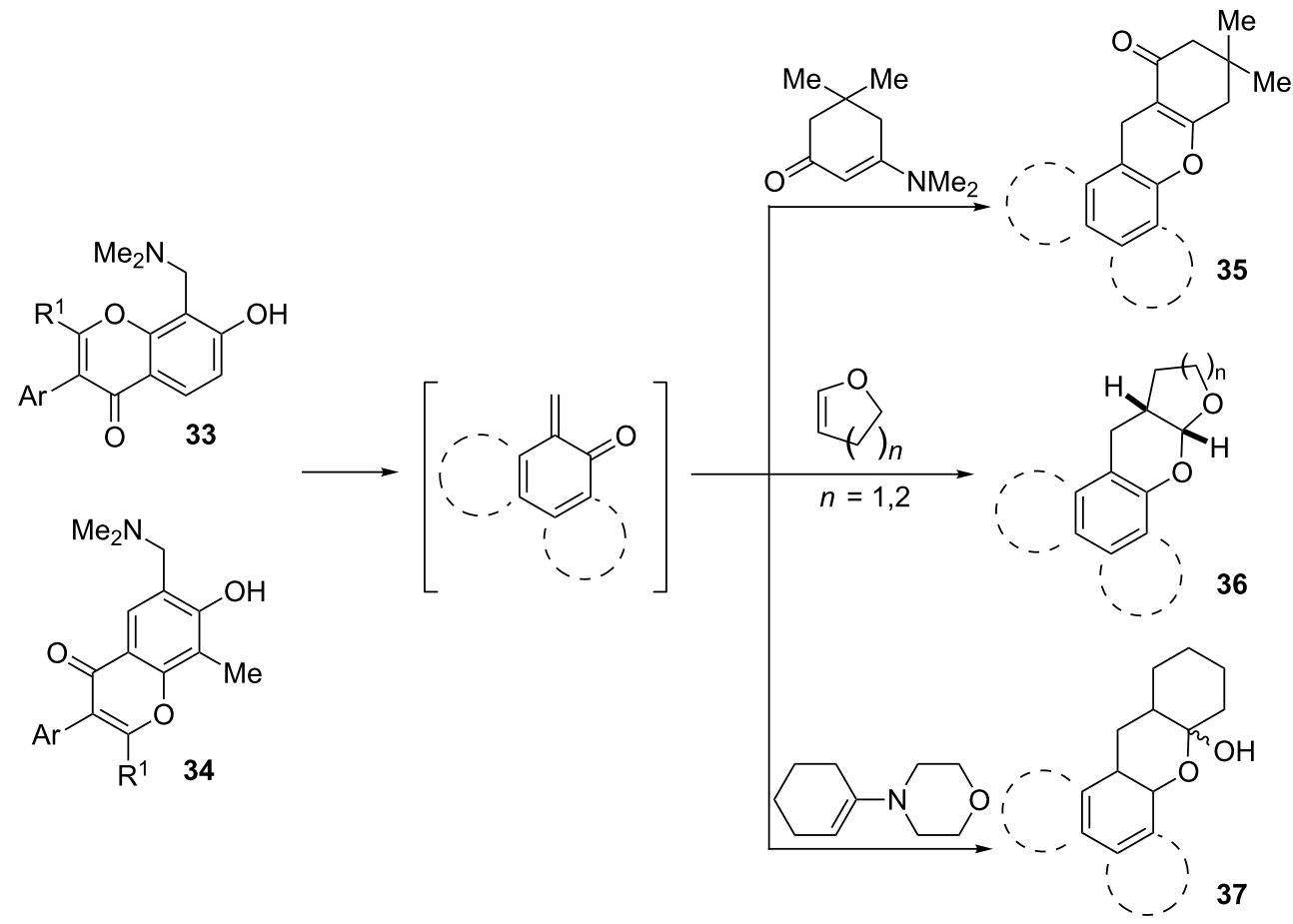

Scheme 4: Cycloadditions of isoflavonoid-derived o-QMs and various dienophiles.

Table 4: Dimerization of $o-Q M s$.

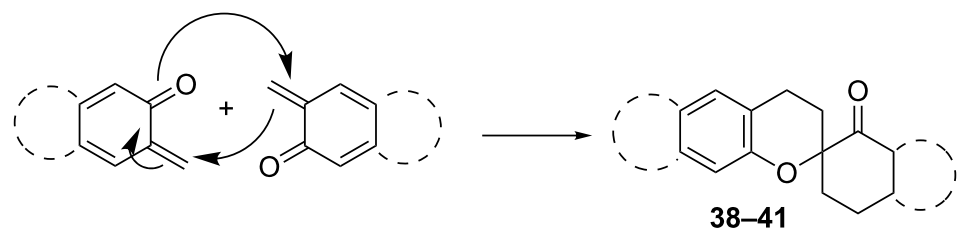

[7]


3<smiles>C=C1C(=O)C=Cc2ccccc21</smiles>

4<smiles>C=C1C=C(OC)C(C(C)C)=C(OC)C1=O</smiles><smiles>O=C1C=Cc2ccccc2C12CCc1c(ccc3ccccc13)O2</smiles>

40<smiles></smiles>

mal desamination of the initial compounds [78]. However, some of the publications report this phenomenon as an advantageous reaction rather than the formation of unexpected side products. As mentioned above [71], Osyanin et al. reported the synthesis of Uvaria scheffleri alkaloid ( \pm )-schefflone. In this publication, the dimerization of the $o$-QM resulting in intermediate $\mathbf{4 1}$ is a key step in the synthesis of the final natural trimer compound.

\section{Reactions with $\mathrm{C}=\mathrm{N}$ dienophiles}

The preparation of novel $o$-QM-condensed poliheterocycles is a relatively new area of Mannich base chemistry. Our research group has also been interested in cycloaddition reactions of $o$-QMs generated from Mannich adducts 42, when a serendipitous reaction occurred. Namely, the formation of new naphthoxazino-isoquinoline derivatives $\mathbf{4 3}$ under neat conditions staring from 1-aminoalkyl-2-naphthols and 6,7-dimethoxy-3,4-dihydroisoquinoline was observed [79]. At the same time, Osyanin et al. reported the same reaction extended by various substituted aminonaphthols [80]. Achieving the syntheses in ethanol at $78{ }^{\circ} \mathrm{C},[4+2]$ cycloaddition took place between the $o$-QM generated from the corresponding aminonaphthol as diene component and cyclic imines playing the role of heterodienophiles (Scheme 5).

Fülöp and co-workers then extended their studies by applying both 2-aminoalkyl-1-naphthols and 1-aminoalkyl-2-naphthols [81]. These bifunctional compounds were reacted with various cyclic imines such as 4,5-dihydrobenzo[c]azepine or 6,7-dihydrothieno[3,2-c]pyridine to have new naphthoxazinobenzazepine $\mathbf{4 4}$ and -thienopyridine $\mathbf{4 5}$ derivatives [82]. Transformations at $80{ }^{\circ} \mathrm{C}$ in 1,4-dioxane as solvent were performed in a microwave reactor to utilize the advantages of this method. As expected, reaction times shortened, while the products were isolated in higher yields in comparison with those found by conventional heating.
The application of $(4 \mathrm{a} S, 8 \mathrm{a} S)$-hexahydroquinoxalin-2-one served as the first example with respect to the use of an enantiomeric cyclic imine in this type of reaction [83]. The formation of the possible naphthoxazino-quinoxalinone diastereomers 46 was investigated and studied by theoretical calculations (Scheme 5). In this and all previous cases, the conformational behaviour of the polyheterocycles formed was also described.

The [4 + 2] cycloadditions between cyclic imines and $o$-QMs derived from Mannich bases could also be successfully applied in the syntheses of natural alkaloid-like compounds 47. Osyanin et al. reported the preparation of rutaecarpine and evodiamine, the 14-oxa analogues of Evodia rutaecarpa alkaloids, starting from 6-methoxy-4,9-dihydro- $\beta$-carboline and various substituted 1-aminoalkyl-2-naphthols [84] (Scheme 5).

\section{Reactions with electron rich aromatic compounds}

The formation of aza-o-QMs is also possible, if the initial phenolic Mannich base bears an aromatic moiety on its benzylic carbon atom. Rueping et al. recently performed reactions between aza-o-QMs in situ generated from $\alpha$-substituted orthoamino benzyl alcohols $\mathbf{4 8}$ and substituted indoles catalysed by $N$-triflylphosphoramides (NTPAs) [85]. (Scheme 6) The process provided new $\mathrm{C}-2$ and $\mathrm{C}$-3-functionalized indole polyheterocycles 49 and 50 in good yields with $90-99 \%$ ee.

One of the latest publications around this topic has been reported by Deb et al. [86,87]. Various 2-(aminoalkyl)phenols or 1-(aminoalkyl)naphthols 51 were reacted with indoles under Brønsted acid catalysis resulting in 3-( $\alpha, \alpha$-diarylmethyl)indoles 52. Then, through $\mathrm{C}-2$ cyclization of the indole ring using $I_{2}$ as catalyst and tert-butyl hydroperoxide as oxidant, chromeno[2,3b]indoles were isolated in 71-98\% yields. In a different reaction pathway, starting from 3-(aminoalkyl)indoles $\mathbf{5 3}$ and phenols or naphthols, 3-( $\alpha, \alpha$-diarylmethyl)indoles 52 were also 


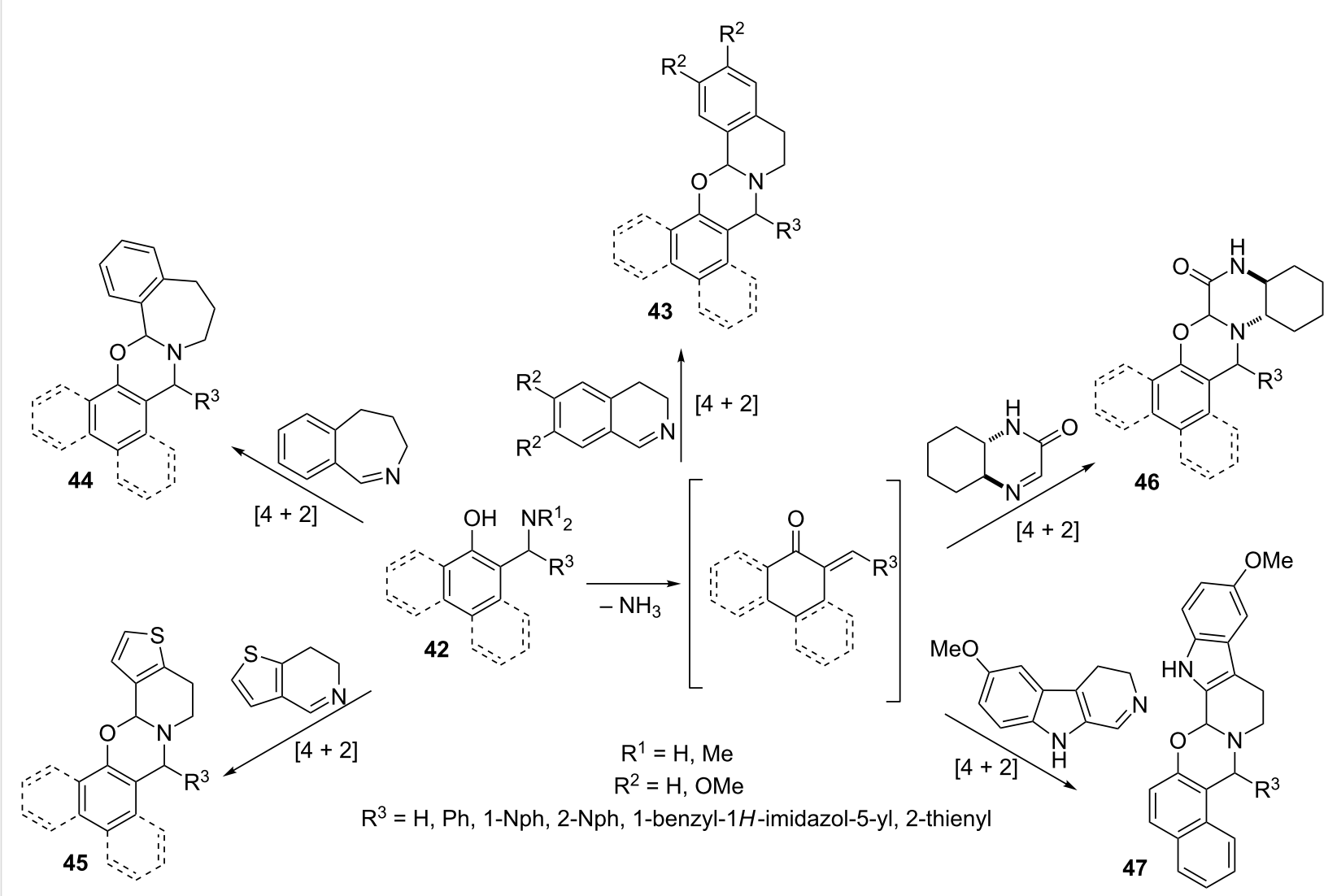

Scheme 5: $[4+2]$ Cycloaddition reactions between aminonaphthols and cyclic amines.

formed in around $90 \%$ yields under microwave irradiation (Scheme 7).

\section{Miscellaneous reactions}

It is also known that $o$-QMs could cross-link two biologically important molecules such as peptides, proteins or nucleic bases. (see section Biological properties) Achieving the reaction under physiological conditions, it is possible to extend the syntheses to biomolecular applications.

Starting from (2-hydroxybenzyl)trimethylammonium iodide (54), Freccero et al. successfully trapped $o$-QMs formed by

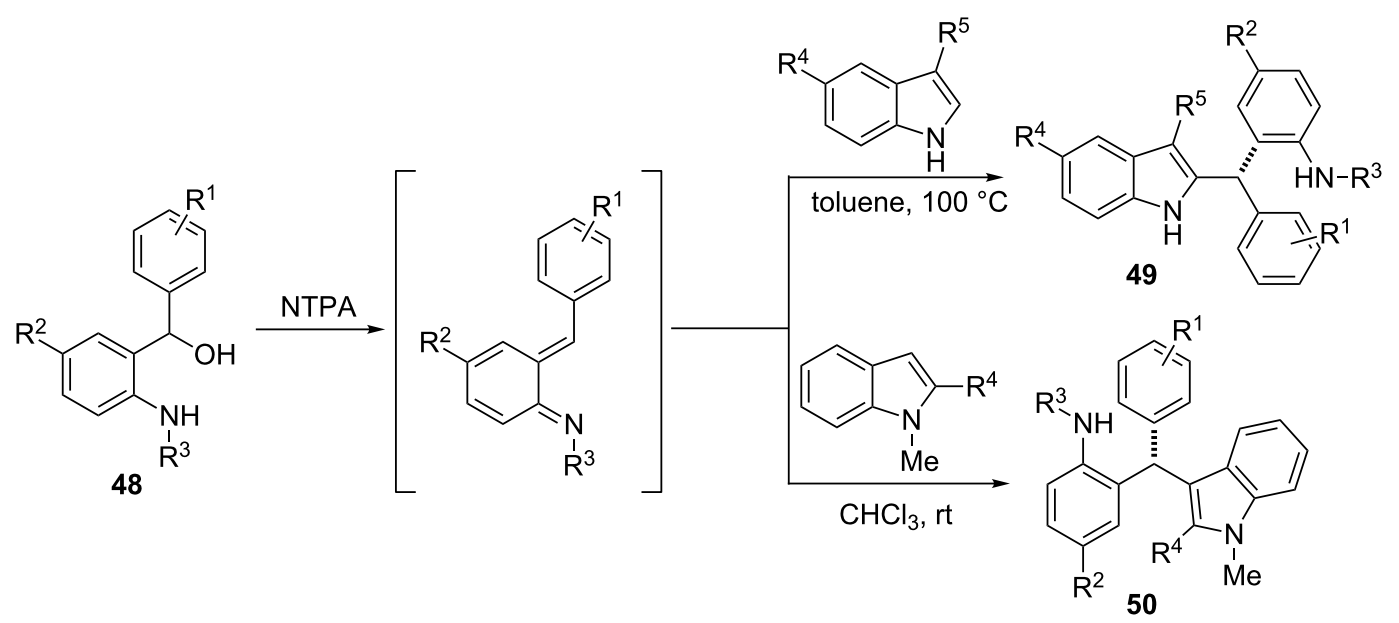

Scheme 6: Brønsted acid-catalysed reaction between aza-o-QMs and 2- or 3-substituted indoles. 


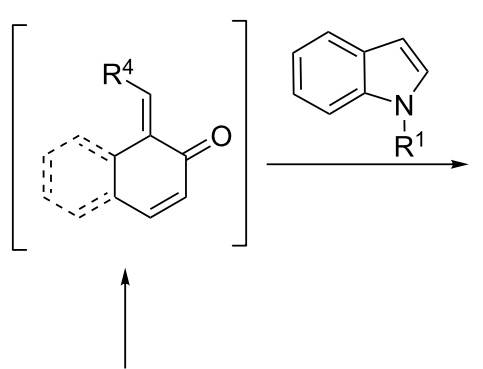<smiles>[R]C(c1c(O)ccc2ccccc12)N(C)C</smiles><smiles>[R3]c1ccc2ccc(C([R])c3cccc4cccc(O)c34)c([R])c2n1</smiles><smiles>Cc1ccc2cc(O)ccc2c1C</smiles><smiles>[R]C=C1C([R])=Nc2ccc([R3])cc21</smiles><smiles>C1CCCCC1</smiles><smiles>[R3]c1ccc2c(c1)c(C([R1])N(C)C)c([R])n2[R1]</smiles>

Scheme 7: Formation of 3-( $\alpha, \alpha-$ diarylmethyl)indoles $\mathbf{5 2}$ in different synthetic pathways.

several N-, O- and S-nucleophiles [88]. They examined both thermal and photochemical generations of such intermediates. By selecting the appropriate reaction conditions (various $\mathrm{pH}$ and temperatures), they were able to alkylate free amino acids, e.g., glycine (Gly), L-serine (Ser), L-cysteine (Cys), L-lysine (Lys), L-tyrosine (Tyr) and glutathione (Glu) in aqueous solution to isolate $\mathbf{5 5}$ (Scheme 8).
Rokita et al. focused on generating $o$-QMs and used them as cross-linking and DNA alkylating agents. Starting from Mannich base 56 and transforming it by a number of synthetic steps, they were managed to elaborate a process that provides easy access to $o$-QM precursors containing a broad array of linkers 57, which were used to connect with site-directing ligands [89] (Scheme 9).

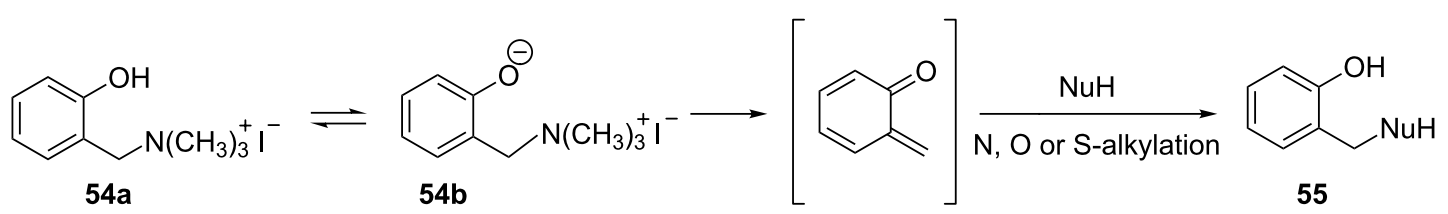

$\mathrm{NuH}=n-\mathrm{PrNH}_{2}, t-\mathrm{BuNH}_{2}$, aniline, piperidine, morpholine, 2-mercaptoethanol, Gly, Ser, Cys, Lys, Tyr, Glu

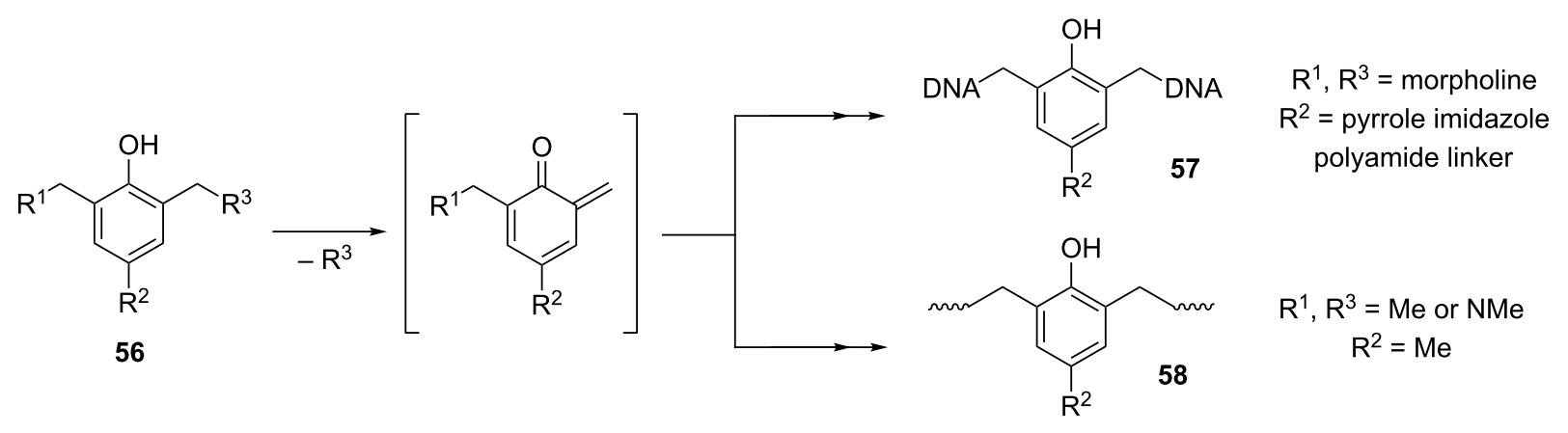


As reactive intermediates, $o$-QMs can also play the role of monomers in polymerization reactions. Ishida et al. reported the ring-opening polymerization of monofunctional alkyl-substituted aromatic amine-based benzoxazines [90]. It was shown that the methylene bridges can be formed by $o$-QMs that are resulted by the cleavage of phenolic Mannich bridge structure 56 (Scheme 9).

\section{Biological properties}

As discussed earlier, $o$-QMs are known as short-lived, highly reactive intermediates. Therefore, their biological activity is mostly examined from the point of view of their application as DNA alkylating agents. One of the first examples was reported by Kearney et al. in 1996 about preformulation studies of the antitumor agent topotecan [91]. The antitumor activity of the compound could be explained by its degradation to highly active zwitterionic species via an $o$-QM intermediate. Dimmock et al. subsequently examined the cytotoxic activity of phenolic azobenzene Mannich bases [92]. Correlations were found between structures and activities against murine P388DI and L1210 cells, human T-lymphocyte cell lines and, in some cases, mutagenous properties were also shown.

Freccero et al. examined the photogeneration by laser flash photolysis and reactivity of naphthoquinone methides as well as their activity as purine selective DNA alkylating agents [93]. Farrell et al. studied the mechanism of the cytotoxic action of naphthoquinone-platinum(II) complexes [94]. Both DNA binding and topoisomerase I inhibition studies proved that the coordination and stabilization of the quinone methide structure can effect marked changes in DNA reactivity. In a recent publication, 3-(aminomethyl)naphthoquinones were investigated from the point of view of cytotoxicity, structure-activity relationships and electrochemical behaviour [95]. Derivatives that contain an aromatic amine and salicylaldehyde or 2-pyridinecarboxaldehyde moieties were found to be the most active against the HL-60 (promyelocytic leukaemia) cell line. Zhou et al. obtained phenolic Mannich bases bearing functional groups that are suitable for cross-linking DNA; therefore, their antitumor effects could also be confirmed [96].

The formation of $o$-QMs and their biological properties were also illustrated by kinetic studies. Rokita et al. using laser flash photolysis showed that formation and reactivity of these intermediates strongly depended on the presence of electron-donating or electron-withdrawing functional groups of the $o-\mathrm{QM}$ precursors [97].

\section{Conclusion}

The high number of publications that has recently appeared on the $o$-QM-mediated Mannich-type transformations is a clear in- dication that the application of this highly-reactive intermediate has made the modified Mannich reaction to be a hot topic again in organic chemistry. This review presents a wide range of applications including cycloadditions and the synthesis of bifunctional amino- or amidonaphthols that can later be transferred as building blocks into several natural or biologically active compounds. Thanks to the immense number of possibilities for Mannich reaction through the use of various amines, aldehydes and electron-rich aromatic compounds, the continued evolution of the literature on these reactions appears to be guaranteed. By the application of various cyclic imines and subsequently extended by the use of nonracemic derivatives, a wide range of enantiomeric polyheterocyclic compounds could be isolated and might be tested as potential anticancer drug candidates.

\section{Acknowledgements}

The authors thank the Ministry of National Economy, National Research Development and Innovation Office [GINOP-2.3.215-2016-00038]; the EU-funded Hungarian Grant [EFOP-3.6.116-2016-00008] and the Hungarian Research Foundation [OTKA No. K115731] for financial support.

\section{References}

1. Bur, S. K.; Martin, S. F. Tetrahedron 2001, 57, 3221-3242. doi:10.1016/S0040-4020(01)00035-7

2. Speckamp, W. N.; Moolenaar, M. J. Tetrahedron 2000, 56, 3817-3856. doi:10.1016/S0040-4020(00)00159-9

3. Arend, M.; Westermann, B.; Risch, N. Angew. Chem., Int. Ed. 1998, 37, 1044-1070. doi:10.1002/(SICI)1521-3773(19980504)37:8<1044::AID-ANIE1044>3. $0 . \mathrm{CO} ; 2-\mathrm{E}$

4. Liras, S.; Davoren, J. E.; Bordner, J. Org. Lett. 2001, 3, 703-706. doi:10.1021/ol0070482

5. Ito, M.; Clark, C. W.; Mortimore, M.; Goh, J. B.; Martin, S. F. J. Am. Chem. Soc. 2001, 123, 8003-8010. doi:10.1021/ja010935v

6. Szatmári, I.; Fülöp, F. Curr. Org. Synth. 2004, 1, 155-165. doi:10.2174/1570179043485402

7. Betti, M. Gazz. Chim. Ital. 1901, 31, 170.

8. Betti, M. Gazz. Chim. Ital. 1901, 31, 191.

9. Betti, M. Organic Syntheses; Collect. Vol. 1; John Wiley \& Sons: New York, 1941; p 381.

10. Szatmári, I.; Fülöp, F. Tetrahedron 2013, 69, 1255-1278. doi:10.1016/j.tet.2012.11.055

11. Van De Water, R. W.; Pettus, T. R. R. Tetrahedron 2002, 58, 5367-5405. doi:10.1016/S0040-4020(02)00496-9

12. Willis, N. J.; Bray, C. D. Chem. - Eur. J. 2012, 18, 9160-9173. doi:10.1002/chem.201200619

13. Bai, W.-J.; David, J. G.; Feng, Z.-G.; Weaver, M. G.; Wu, K.-L.; Pettus, T. R. R. Acc. Chem. Res. 2014, 47, 3655-3664. doi:10.1021/ar500330x

14. Toteva, M. M.; Richard, J. P. Adv. Phys. Org. Chem. 2011, 45, 39-91. doi:10.1016/B978-0-12-386047-7.00002-3

15. Osipov, D. V.; Osyanin, V. A.; Klimochkin, Y. N. Russ. Chem. Rev. 2017, 86, 625-687. doi:10.1070/RCR4679

16. Rokita, S. E., Ed. Quinone Methides; John Wiley \& Sons, Inc.: Hoboken, New Jersey, 2009. doi:10.1002/9780470452882 
17. Singh, M. S.; Nagaraju, A.; Anand, N.; Chowdhury, S. RSC Adv. 2014, 4, 55924-55959. doi:10.1039/C4RA11444B

18. Csütörtöki, R.; Szatmári, I.; Fülöp, F. Curr. Org. Synth. 2013, 10, 564-583. doi:10.2174/1570179411310040004

19. Nasr-Esfahani, M.; Montazerozohori, M.; Taei, M. C. R. Chim. 2016, 19, 986-994. doi:10.1016/j.crci.2016.02.003

20. Zali, A.; Shokrolahi, A. Chin. Chem. Lett. 2012, 23, 269-272. doi:10.1016/j.cclet.2011.12.002

21. Borhade, A. V.; Uphade, B. K.; Tope, D. R. Res. Chem. Intermed. 2014, 40, 211-223. doi:10.1007/s11164-012-0956-4

22. Safari, J.; Zarnnegar, Z. J. Mol. Catal. A: Chem. 2013, 379, 269-276. doi:10.1016/j.molcata.2013.08.028

23. Zolfigol, M. A.; Baghery, S.; Moosavi-Zare, A. R.; Vahdat, S. M.; Alinezhad, H.; Norouzi, M. RSC Adv. 2015, 5, 45027-45037. doi:10.1039/C5RA02718G

24. Hajipour, A. R.; Ghayeb, Y.; Sheikhan, N.; Ruoho, A. E. Tetrahedron Lett. 2009, 50, 5649-5651. doi:10.1016/j.tetlet.2009.07.116

25. Mulla, S. A. R.; Salama, T. A.; Pathan, M. Y.; Inamdar, S. M.; Chavan, S. S. Tetrahedron Lett. 2013, 54, 672-675. doi:10.1016/j.tetlet.2012.12.004

26. Kotadia, D. A.; Soni, S. S. J. Mol. Catal. A: Chem. 2012, 353-354. doi:10.1016/j.molcata.2011.11.003

27. Muskawar, P. N.; Kumar, S. S.; Bhagat, P. R. J. Mol. Catal. A: Chem. 2013, 380, 112-117. doi:10.1016/j.molcata.2013.09.026

28. Safari, J.; Zarnegar, Z. Ultrason. Sonochem. 2014, 21, 1132-1139. doi:10.1016/j.ultsonch.2013.10.024

29. Khodaei, M. M.; Khosropour, A. R.; Morghanian, H. Synlett 2006, 916-920. doi:10.1055/s-2006-939034

30. Kantevari, S.; Vuppalapati, S. V. N.; Nagarapu, L. Catal. Commun. 2007, 8, 1857-1862. doi:10.1016/j.catcom.2007.02.022

31. Patil, S. B.; Singh, P. R.; Surpur, M. P.; Samant, S. D. Synth. Commun. 2007, 37, 1659-1664. doi:10.1080/00397910701263858

32. Das, B.; Laxminarayana, K.; Ravikanth, B.; Rao, B. R. J. Mol. Catal. A: Chem. 2007, 261, 180-183. doi:10.1016/j.molcata.2006.07.077

33. Nagarapu, L.; Baseeruddin, M.; Apuri, S.; Kantevari, S. Catal. Commun. 2007, 8, 1729-1734. doi:10.1016/j.catcom.2007.02.008

34. Mahdavinia, G. H.; Bigdeli, M. A.; Heravi, M. M. Chin. Chem. Lett. 2008, 19, 1171-1174. doi:10.1016/j.cclet.2008.06.048

35. Shaterian, H. R.; Yarahmadi, H.; Ghashang, M. Tetrahedron 2008, 64, 1263-1269. doi:10.1016/j.tet.2007.11.070

36. Das, B.; Kumar, D. N.; Laxminarayana, K.; Ravikanth, B. Helv. Chim. Acta 2007, 90, 1330-1334. doi:10.1002/hlca.200790134

37. Srihari, G.; Nagaraju, M.; Murthy, M. M. Helv. Chim. Acta 2007, 90, 1497-1504. doi:10.1002/hlca.200790156

38. Anary-Abbasinejad, M.; Hassanabadi, A.; Kamali-Gharamaleki, M.; Saidipoor, A.; Anaraki-Ardakani, H. J. Chem. Res. 2007, 644-646. doi:10.3184/030823407X266207

39. Shaterian, H. R.; Yarahmadi, H. ARKIVOC 2008, 105-114. doi:10.3998/ark.5550190.0009.212

40. Shaterian, H. R.; Yarahmadi, H.; Ghashang, M. Bioorg. Med. Chem. Lett. 2008, 18, 788-792. doi:10.1016/j.bmcl.2007.11.035

41. Su, W.; Tang, W.; Li, J. J. Chem. Res. 2008, 123-128. doi:10.3184/030823408X298508

42. Khabazzadeh, H.; Saidi, K.; Seyedi, N. J. Chem. Sci. 2009, 121, 429-433. doi:10.1007/s12039-009-0050-7
43. Mahdavinia, G. H.; Bigdeli, M. A. Chin. Chem. Lett. 2009, 20, 383-386. doi:10.1016/j.cclet.2008.12.018

44. Patil, S. B.; Singh, P. R.; Surpur, M. P.; Samant, S. D. Ultrason. Sonochem. 2007, 14, 515-518. doi:10.1016/j.ultsonch.2006.09.006

45. Nagawade, R. R.; Shinde, D. B. Mendeleev Commun. 2007, 17, 299-300. doi:10.1016/j.mencom.2007.09.018

46. Nandi, G. C.; Samai; Kumar, R.; Singh, M. S. Tetrahedron Lett. 2009, 50, 7220-7222. doi:10.1016/j.tetlet.2009.10.055

47. Shaterian, H. R.; Azizi, K.; Fahimi, N. Chem. Sci. Trans. 2012, 1, 73-84. doi:10.7598/cst2012.139

48. Ghorbani-Vaghei, R.; Malaekehpour, S. M. Cent. Eur. J. Chem. 2010, 8, 1086-1089. doi:10.2478/s11532-010-0077-0

49. Wang, C.; Wan, Y.; Wang, H.-Y.; Zhao, L.-L.; Shi, J.-J.; Zhang, X.-X.; Wu, H. J. Heterocycl. Chem. 2013, 50, 496-500. doi:10.1002/jhet.1124

50. Hazeri, N.; Maghsoodlou, M. T.; Habibi-Khorassani, S. M.; Aboonajmi, J.; Safarzaei, M. Chem. Sci. Trans. 2013, 2, S330-S336. doi:10.7598/cst2013.426

51. Singh, R. K.; Duvedi, R. Arabian J. Chem. 2018, 11, 91-98. doi:10.1016/j.arabjc.2014.08.022

52. Li, T.; Zhai, X.; Singh, D.; Singh, R. K.; Xu, X. Asian J. Chem. 2014, 26, 5207-5211. doi:10.14233/ajchem.2014.16707

53. Davoodnia, A.; Mahjoobin, R.; Tavakoli-Hoseini, N. Chin. J. Catal. 2014, 35, 490-495. doi:10.1016/S1872-2067(14)60011-5

54. Shaikh, K. A.; Chaudhar, U. N.; Ningdale, V. B. IOSR J. Appl. Chem. 2014, 7, 90-93. doi:10.9790/5736-07429093

55. Singh, R. K.; Singh, B.; Duvedi, R.; Kumar, S. Res. Chem. Intermed. 2015, 41, 4083-4099. doi:10.1007/s11164-013-1513-5

56. Wang, M.; Liang, Y.; Zhang, T. T.; Gao, J. J. Chin. Chem. Lett. 2012, 23, 65-68. doi:10.1016/j.cclet.2011.10.008

57. Niralwad, K. S.; Shingate, B. B.; Shingare, M. S. Chin. Chem. Lett. 2011, 22, 551-554. doi:10.1016/j.cclet.2010.11.018

58. Hashemi, H.; Sardarian, A. R. J. Iran. Chem. Soc. 2013, 10, 745-750. doi:10.1007/s13738-012-0208-y

59. Rani,, G. S.; Reddy, V. K. S.; Prasad, R. B. N.; Devi, B. L. A. P. Int. J. Adv. Res. Chem. Sci. 2015, 2, 22-30.

60. Hajjami, M.; Ghorbani, F.; Bakhti, F. Appl. Catal., A 2014, 470 , 303-310. doi:10.1016/j.apcata.2013.11.002

61. Moghanian, H.; Ebrahimi, S. J. Saudi Chem. Soc. 2014, 18, 165-168. doi:10.1016/j.jscs.2011.06.017

62. Forouzani, M.; Ghasemnejad-Bosra, H. Arabian J. Chem. 2016, 9 , S752-S755. doi:10.1016/j.arabjc.2011.08.002

63. El Kaïm, L.; Grimaud, L.; Oble, J. Org. Biomol. Chem. 2006, 4, 3410-3413. doi:10.1039/B610229H

64. Mudududdla, R.; Jain, S. K.; Bharate, J. B.; Gupta, A. P.; Singh, B.; Vishwakarma, R. A.; Bharate, S. B. J. Org. Chem. 2012, 77, 8821-8827. doi:10.1021/jo3017132

65. Sharma, R.; Abbat, S.; Mudududdla, R.; Vishwakarma, R. A.; Bharatam, P. V.; Bharate, S. B. Tetrahedron Lett. 2015, 56, 4057-4059. doi:10.1016/j.tetlet.2015.04.072

66. Cherkadu, V.; Kalavagunta, P. K.; Ravirala, N.; Shivananju, N. S.; Priya, B. S. Synlett 2016, 27, 2795-2798. doi:10.1055/s-0036-1588595

67. Osyanin, V. A.; Osipov, D. V.; Klimochkin, Y. N. Tetrahedron 2012, 68, 5612-5618. doi:10.1016/j.tet.2012.04.065

68. Nakatani, K.; Higashida, N.; Saito, I. Tetrahedron Lett. 1997, 38, 5005-5008. doi:10.1016/S0040-4039(97)01071-X

69. Pettigrew, J. D.; Freeman, R. P.; Wilson, P. D. Can. J. Chem. 2004, 82, 1640-1648. doi:10.1139/v04-138

70. Osipov, D. V.; Osyanin, V. A.; Klimochkin, Y. N. Synlett 2012, 917-919. doi:10.1055/s-0031-1290611 
71. Osyanin, V. A.; Ivleva, E. A.; Klimochkin, Y. N. Synth. Commun. 2012, 42, 1832-1847. doi:10.1080/00397911.2010.545164

72. Osyanin, V. A.; Osipov, D. V.; Klimochkin, Y. N. J. Org. Chem. 2013, 78, 5505-5520. doi:10.1021/jo400621r

73. Willis, N. J.; Bray, C. D. RSC Adv. 2015, 5, 80212-80215. doi:10.1039/C5RA17108C

74. Huang, Y.; Hayashi, T. J. Am. Chem. Soc. 2015, 137, 3201. doi:10.1021/jacs.5b00216

75. Huang, P.-J. J.; Cameron, T. S.; Jha, A. Tetrahedron Lett. 2009, 50, 51-54. doi:10.1016/j.tetlet.2008.10.083

76. Frasinyuk, M. S.; Mrug, G. P.; Bondarenko, S. P.; Khilya, V. P.; Sviripa, V. M.; Syrotchuk, O. A.; Zhang, W.; Cai, X.; Fiandalo, M. V.; Mohler, J. L.; Liu, C.; Watt, D. S. ChemMedChem 2016, 11, 600-611. doi:10.1002/cmdc.201600008

77. Schenck, L. W.; Kuna, K.; Frank, W.; Albert, A.; Asche, C.; Kucklaender, U. Bioorg. Med. Chem. 2006, 14, 3599-3614. doi:10.1016/j.bmc.2006.01.026

78. Bilgiç, S.; Bilgiç, O.; Büyükkidan, B.; Gündüz, M. J. Chem. Res. 2007, 76-79. doi:10.3184/030823407X198131

79. Szatmári, I.; Fülöp, F. Tetrahedron Lett. 2011, 52, 4440-4442. doi:10.1016/j.tetlet.2011.06.074

80. Osyanin, V. A.; Ivleva, E. A.; Osipov, D. V.; Klimochkin, Y. N. Chem. Heterocycl. Compd. 2011, 47, 845-850. doi:10.1007/s10593-011-0845-6

81. Szatmári, I.; Hexdenreich, M.; Koch, A.; Fülöp, F.; Kleinpeter, E. Tetrahedron 2013, 69, 7455-7465. doi:10.1016/j.tet.2013.06.094

82. Szatmári, I.; Barta, P.; Csámpai, A.; Fülöp, F. Tetrahedron 2017, 73, 4790-4804. doi:10.1016/j.tet.2017.06.060

83. Szatmári, I.; Barta, P.; Tóth, G.; Balázs, A.; Halász, J.; Fülöp, F. Eur. J. Org. Chem. 2017, 5537-5545. doi:10.1002/ejoc.201700699

84. Osyanin, V. A.; Ivleva, E. A.; Klimochkin, Y. N. Chem. Heterocycl. Compd. 2012, 48, 795-798. doi:10.1007/s10593-012-1058-3

85. Liao, H.-H.; Chatupheeraphat, A.; Hsiao, C.-C.; Atodiresei, I.; Rueping, M. Angew. Chem., Int. Ed. 2015, 54, 15540-15544. doi:10.1002/anie.201505981

86. Deb, M. L.; Dev Pegu, C.; Deka, B.; Dutta, P.; Kotmale, A. S.; Baruah, P. K. Eur. J. Org. Chem. 2016, 3441-3448. doi:10.1002/ejoc.201600546

87. Deb, M. L.; Das, C.; Deka, B.; Saikia, P. J.; Baruah, P. K. Synlett 2016, 27, 2788-2794. doi:10.1055/s-0036-1588887

88. Modica, E.; Zanaletti, R.; Freccero, M.; Mella, M. J. Org. Chem. 2001, 66, 41-52. doi:10.1021/jo0006627

89. Kumar, D.; Rokita, S. E. Tetrahedron Lett. 2004, 45, 2887-2889. doi:10.1016/j.tetlet.2004.02.070

90. Ishida, H.; Sanders, D. P. Polymer 2001, 42, 3115-3125. doi:10.1016/S0032-3861(00)00498-5

91. Kearney, A. S.; Patel, K.; Palepu, N. R. Int. J. Pharm. 1996, 127, 229-237. doi:10.1016/0378-5173(95)04218-0

92. Dimmock, J. R.; Erciyas, E.; Kumar, P.; Hetherington, M.; Quail, J. W.; Pugazhenthi, U.; Arpin, S. A.; Hayes, S. J.; Allen, T. M.; Halleran, S.; De Clercq, E.; Balzarini, J.; Stables, J. P. Eur. J. Med. Chem. 1997, 32, 583-594. doi:10.1016/S0223-5234(97)83284-9

93. Verga, D.; Nadai, M.; Doria, F.; Percivalle, C.; Di Antonio, M.; Palumbo, M.; Richter, S. N.; Freccero, M. J. Am. Chem. Soc. 2010, 132, 14625-14637. doi:10.1021/ja1063857

94. Neves, A. P.; Pereira, M. X. G.; Peterson, E. J.; Kipping, R.; Vargas, M. D.; Silva, F. P., Jr.; Carneiro, J. W. M.; Farrell, N. P. J. Inorg. Biochem. 2013, 119, 54-64. doi:10.1016/j.jinorgbio.2012.10.007
95. Da Silva, G. B.; Neves, A. P.; Vargas, M. D.; Marinho-Filho, J. D. B.; Costa-Lotufo, L. V. Bioorg. Med. Chem. Lett. 2016, 26, 3537-3542. doi:10.1016/j.bmcl.2016.06.027

96. Zhang, L.; Ren, L.; Minghui, B.; Weng, L.; Huang, J.; Wu, L.; Deng, M.; Zhou, X. Bioorg. Med. Chem. 2007, 15, 6920-6926. doi:10.1016/j.bmc.2007.07.053

97. Weinert, E. E.; Dondi, R.; Colloredo-Melz, S.; Frankenfield, K. N.; Mitchell, C. H.; Freccero, M.; Rokita, S. E. J. Am. Chem. Soc. 2006, 128, 11940-11947. doi:10.1021/ja062948k

\section{License and Terms}

This is an Open Access article under the terms of the Creative Commons Attribution License

(http://creativecommons.org/licenses/by/4.0), which permits unrestricted use, distribution, and reproduction in any medium, provided the original work is properly cited.

The license is subject to the Beilstein Journal of Organic Chemistry terms and conditions: (https://www.beilstein-journals.org/bjoc)

The definitive version of this article is the electronic one which can be found at: doi:10.3762/bjoc. 14.43 